\title{
Lens spaces, rational balls and the ribbon conjecture
}

\author{
PAOLO LisCA
}

\begin{abstract}
We apply Donaldson's theorem on the intersection forms of definite 4-manifolds to characterize the lens spaces which smoothly bound rational homology 4-dimensional balls. Our result implies, in particular, that every smoothly slice 2-bridge knot is ribbon, proving the ribbon conjecture for 2 -bridge knots.
\end{abstract}

$57 \mathrm{M} 25$

\section{Introduction}

It is a well-known fact that every ribbon knot is smoothly slice. The ribbon conjecture states that, conversely, a smoothly slice knot is ribbon. In this paper we prove that the ribbon conjecture holds for 2-bridge knots, deducing this result from a characterization of the 3-dimensional lens spaces which smoothly bound rational homology 4-dimensional balls (Theorem 1.2 below).

A link in $S^{3}$ is called 2-bridge if it can be isotoped until it has exactly two local maxima with respect to a standard height function. Figure 1 represents the 2-bridge link $L\left(c_{1}, \ldots, c_{n}\right)$, where $c_{i} \in \mathbb{Z}, i=1, \ldots, n$. Given coprime integers $p>q>0$ with

$$
\frac{p}{q}=c_{1}+\frac{1}{c_{2}+\frac{1}{\ddots+\frac{1}{c_{n}}}}, \quad c_{i}>0 \text { for } i=1, \ldots, n,
$$

the 2-bridge link $K(p, q)$ is, by definition, $L\left(c_{1}, \ldots, c_{n}\right)$. When $p$ is even, $K(p, q)$ is a 2-component link, when $p$ is odd $K(p, q)$ is a knot. It is well-known (see, for example, Burde and Zieschang [1, Chapter 12]) that $K(p, q)$ and $K\left(p^{\prime}, q^{\prime}\right)$ are isotopic if and only if $p=p^{\prime}$ and either $q=q^{\prime}$ or $q q^{\prime} \equiv 1(\bmod p)$, and that every 2-bridge

link is isotopic to some $K(p, q)$. Moreover, $K(p, p-q)$ is isotopic to the mirror image of $K(p, q)$.

Now we recall what is known about 2-bridge knots with regard to the ribbon conjecture. In order to do that, the following definition is needed. 

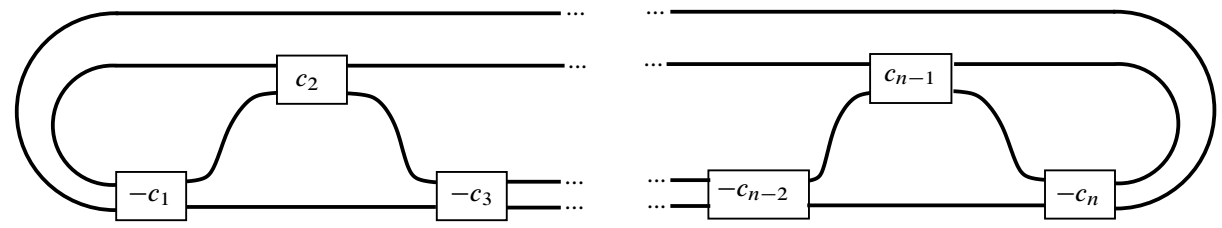

$(n$ odd $)$

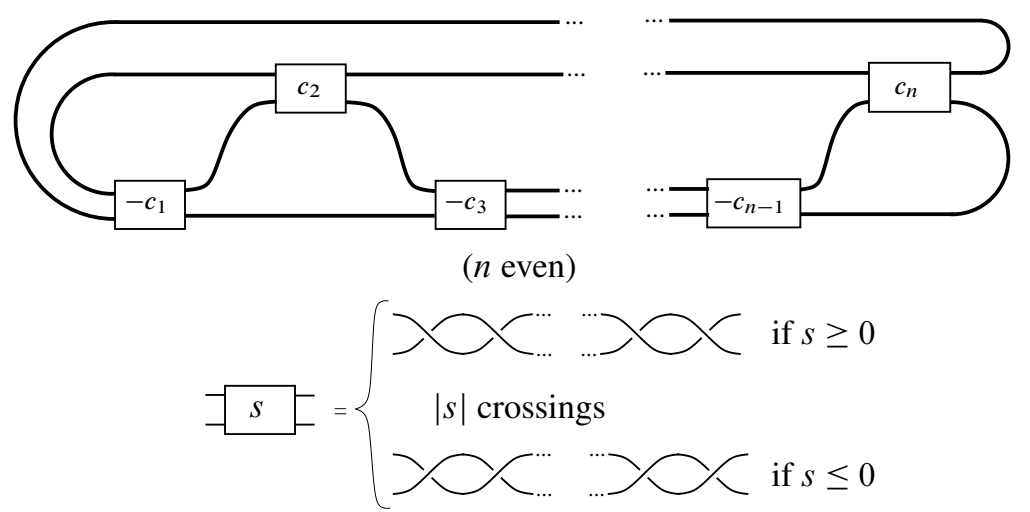

Figure 1: The 2-bridge link $L\left(c_{1}, \ldots, c_{n}\right)$

Definition 1.1 Let $\mathbb{Q}_{>0}$ denote the set of positive rational numbers, and define maps $f, g: \mathbb{Q}_{>0} \rightarrow \mathbb{Q}_{>0}$ by setting, for $\frac{p}{q} \in \mathbb{Q}_{>0}, p>q>0,(p, q)=1$,

$$
f\left(\frac{p}{q}\right)=\frac{p}{p-q}, \quad g\left(\frac{p}{q}\right)=\frac{p}{q^{\prime}},
$$

where $p>q^{\prime}>0$ and $q q^{\prime} \equiv 1(\bmod p)$. Define $\mathcal{R} \subset \mathbb{Q}_{>0}$ to be the smallest subset of $\mathbb{Q}_{>0}$ such that $f(\mathcal{R}) \subseteq \mathcal{R}, g(\mathcal{R}) \subseteq \mathcal{R}$ and $\mathcal{R}$ contains the set of rational numbers $\frac{p}{q}$ such that $p>q>0,(p, q)=1, p=m^{2}$ for some $m \in \mathbb{N}$ and $q$ is of one of the following types:

(1) $m k \pm 1$ with $m>k>0$ and $(m, k)=1$;

(2) $d(m \pm 1)$, where $d>1$ divides $2 m \mp 1$ and

(3) $d(m \pm 1)$, where $d>1$ is odd and divides $m \pm 1$.

According to Siebenmann [11], Casson, Gordon and Conway showed that every knot of the form $K(p, q)$ with $\frac{p}{q} \in \mathcal{R}$ is ribbon. The interior of any ribbon disk can be radially pushed inside the 4-ball $B^{4}$ to obtain a smoothly embedded disk, and the 2-fold cover of $B^{4}$ branched along a slicing disk for $K(p, q)$ is a smooth rational homology ball with boundary the lens space $L(p, q)$. Therefore if $K(p, q)$ is a knot (ie if $p$ is odd) 
we have the following implications:

$$
\frac{p}{q} \in \mathcal{R} \Rightarrow K(p, q) \text { ribbon } \Rightarrow K(p, q) \text { smoothly slice } \Rightarrow L(p, q)=\partial W,
$$

where $W$ is a smooth 4 -manifold with $H_{*}(W ; \mathbb{Q}) \cong H_{*}\left(B^{4} ; \mathbb{Q}\right)$. Casson and Gordon [2] observed that if $K(p, q)$ is a smoothly slice knot then $p$ is a perfect square. Moreover, they proved that if the 2-bridge knot $K\left(\mathrm{~m}^{2}, q\right)$ is ribbon then

$$
\frac{2}{m^{2}} \sum_{s=1}^{m^{2}-1} \cot \left(\frac{\pi s}{m^{2}}\right) \cot \left(\frac{\pi q s}{m^{2}}\right) \sin ^{2}\left(\frac{\pi r s}{m}\right)= \pm 1, \quad r=1, \ldots, m-1 .
$$

Casson and Gordon [2, page 188] used (1-1) to show that if a 2-bridge knot $K\left(m^{2}, q\right)$ is ribbon and $m \leq 105$ then $\frac{m^{2}}{q}$ belongs to $\mathcal{R}$. Fintushel and Stern [5, Theorem 6.1] proved that (1-1) hold under the assumption that $L\left(m^{2}, q\right)$ is the boundary of a smooth rational homology ball $W$ with $H_{2}(W ; \mathbb{Z})$ without 2-torsion. In [7] Owens and Strle used a result by Oszváth and Szabó [8, Theorem 9.6] to find a priori different obstructions for $K\left(m^{2}, q\right)$ to be smoothly slice, and verified that for $m \leq 105$ these new obstructions give the same constraints as (1-1). It is not known whether (1-1) imply that the knot $K\left(m^{2}, q\right)$ is smoothly slice.

The following is our main result.

Theorem 1.2 Let $p>q>0$ be coprime integers. Then, the following statements are equivalent.

(1) The lens space $L(p, q)$ smoothly bounds a rational homology ball.

(2) There exist:

(a) A surface with boundary $\Sigma$, homeomorphic to a disk if $p$ is odd and to the disjoint union of a disk and a Möbius band if $p$ is even and

(b) A ribbon immersion $i: \Sigma \uparrow \rightarrow S^{3}$ with $i(\partial \Sigma)=K(p, q)$.

(3) $\frac{p}{q}$ belongs to $\mathcal{R}$.

Theorem 1.2 immediately implies the following result, which settles the ribbon conjecture for 2-bridge knots.

Corollary 1.3 Let $p>q>0$ be coprime integers with $p$ odd. Then, the following statements are equivalent:

(1) $\frac{p}{q}$ belongs to $\mathcal{R}$;

(2) $K(p, q)$ is a ribbon knot;

(3) $K(p, q)$ is a smoothly slice knot and 
(4) $L(p, q)$ smoothly bounds a rational homology ball.

In particular, the ribbon conjecture holds for 2-bridge knots.

The proof of Theorem 1.2 is based on the following simple idea. If a lens space $L(p, q)$ smoothly bounds a rational homology ball $W(p, q)$, one can form a smooth negative definite 4-manifold $X(p, q)$ by taking the union of $-W(p, q)$ with a canonical 4dimensional plumbing $P(p, q)$ bounding $L(p, q)$. Since $X(p, q)$ is negative definite, Donaldson's celebrated theorem [4] implies that the intersection lattice $Q_{X(p, q)}$ of $X(p, q)$ is isomorphic to the standard diagonal intersection lattice $\mathbf{D}^{n}$, where $n=$ $b_{2}(X(p, q))$. Therefore there is an embedding of intersection lattices $Q_{P(p, q)} \hookrightarrow \mathbf{D}^{n}$, and since $-L(p, q)=L(p, p-q)$ smoothly bounds the rational homology ball $-W(p, q)$, for some $n^{\prime}$ there is an embedding $Q_{P(p, p-q)} \hookrightarrow \mathbf{D}^{n^{\prime}}$ as well. The existence of both embeddings (it is easy to see that a single embedding is not enough) gives constraints on the pair $(p, q)$ which eventually lead to the proof of Theorem 1.2. In spite of the simplicity of this idea, the algebro-combinatorial machinery we must set up to work out the above constraints is fairly complex and occupies Sections 2-7 of the paper. Here is the gist of what we do. We can write

$$
\frac{p}{q}=a_{1}-\frac{1}{a_{2}-\frac{1}{\ddots \cdot-\frac{1}{a_{n}}}}, \quad \frac{p}{p-q}=b_{1}-\frac{1}{b_{2}-\frac{1}{\ddots-\frac{1}{b_{n^{\prime}}}}},
$$

for some integers $a_{i}, b_{j} \geq 2$ for $i=1, \ldots, n, j=1, \ldots, n^{\prime}$. It turns out (see Lemma 2.6) that

$$
\sum_{i=1}^{n}\left(a_{i}-3\right)+\sum_{j=1}^{n^{\prime}}\left(b_{j}-3\right)=-2,
$$

therefore, up to replacing $(p, q)$ with $(p, p-q)$, we may assume

$$
\sum_{i=1}^{n}\left(a_{i}-3\right)<0 .
$$

A suitable set of generators for $H_{2}(P(p, q) ; \mathbb{Z})$ together with the embedding $Q_{P(p, q)} \hookrightarrow$ $\mathbf{D}^{n}$ give rise to a subset $S=\left\{v_{1}, \ldots, v_{n}\right\} \subset \mathbf{D}^{n}$ with

$$
v_{i} \cdot v_{j}= \begin{cases}-a_{i} & \text { if }|i-j|=0, \\ 1 & \text { if }|i-j|=1, \\ 0 & \text { if }|i-j|>1 .\end{cases}
$$


We call such subsets standard. In Sections 2-7 we study the standard subsets of $\mathbf{D}^{n}$ satisfying (1-2). In Section 7 we show that the string of integers $\left(a_{1}, \ldots, a_{n}\right)$ associated to such a subset must belong (for a fixed $n$ ) to a finite list which we describe explicitly. This gives the constraints mentioned above. In Section 8 we prove the existence of ribbon surfaces for all the links required by Theorem $1.2^{1}$, and in Section 9 we prove Theorem 1.2 using all the results obtained in the previous sections. Each section starts with a brief outline summarizing its purpose, contents and relationships with the other sections.

\section{Acknowledgments}

The author is grateful to Andrew Casson for generous help, to Cameron Gordon for informative e-mail correspondence and to the anonymous referee for useful comments.

\section{First definitions and preliminary results}

Outline In this section we introduce definitions which will be used throughout the paper. In particular, the concept of good subset (see Definition 2.2) is crucial in Section 3, Section 4 and Section 5, while the invariant $I(S)$ (see Definition 2.3) is the key quantity on which the proof of Theorem 1.2 is based. We also prove Lemma 2.4, which is the basis of the inductive process used in the subsequent sections, and Lemma 2.6, which will be directly quoted in the proof of Theorem 1.2 in Section 9.

Let $\mathbf{D}$ denote the intersection lattice $(\mathbb{Z},(-1))$, and let $\mathbf{D}^{n}$ be the orthogonal direct sum of $n$ copies of $\mathbf{D}$. Fix generators $e_{1}, \ldots, e_{n} \in \mathbf{D}^{n}$ such that

$$
e_{i} \cdot e_{j}=-\delta_{i j}, \quad i, j=1, \ldots, n .
$$

Observe that the group of automorphisms $\operatorname{Aut}\left(\mathbf{D}^{n}\right)$ contains the reflections across each hyperplane orthogonal to an $e_{i}$ as well as the all the transformations determined by the permutations of $\left\{e_{1}, \ldots, e_{n}\right\}$. Given a subset $S=\left\{v_{1}, \ldots, v_{n}\right\} \subseteq \mathbf{D}^{n}$, we define

$$
\begin{aligned}
E_{i}^{S}: & =\left\{j \in\{1, \ldots, n\} \mid v_{j} \cdot e_{i} \neq 0\right\}, \quad i=1, \ldots, n, \\
V_{i} & :=\left\{j \in\{1, \ldots, n\} \mid e_{j} \cdot v_{i} \neq 0\right\}, \quad i=1, \ldots, n,
\end{aligned}
$$

and

$$
p_{i}(S):=\left|\left\{j \in\{1, \ldots, n\}|| E_{j}^{S} \mid=i\right\}\right|, \quad i=1, \ldots, n .
$$

\footnotetext{
${ }^{1}$ The results of Section 8 were known previously for knots [11] (although even in the case of knots we were unable to recover all of them from [11]). In Section 8 we give a self-contained account valid for links and adapted to our conventions.
} 
Let $v_{1}, \ldots, v_{n} \in \mathbf{D}^{n}$ be elements such that, for $i, j \in\{1, \ldots, n\}$,

$$
v_{i} \cdot v_{j}= \begin{cases}-a_{i} \leq-2 & \text { if } i=j, \\ 0 \text { or } 1 & \text { if }|i-j|=1, \\ 0 & \text { if }|i-j|>1\end{cases}
$$

for some integers $a_{i}, i=1, \ldots, n$.

Remark 2.1 Elements $v_{1}, \ldots, v_{n} \in \mathbf{D}^{n}$ satisfying Conditions (2-1) are linearly independent over $\mathbb{Z}$. In fact, it is easy to check that the associated intersection matrix

$$
Q:=\left(v_{i} \cdot v_{j}\right)
$$

is nonsingular. The independence of $v_{1}, \ldots, v_{n}$ follows immediately from the fact that

$$
Q=-M M^{t},
$$

where $M:=\left(m_{i j}\right)$ is defined by $v_{i}=\sum_{j} m_{i j} e_{j}$.

Let $S=\left\{v_{1}, \ldots, v_{n}\right\} \subseteq \mathbf{D}^{n}$ be a subset which satisfies (2-1). We define the intersection graph of $S$ as the graph having as vertices $v_{1}, \ldots, v_{n}$, and an edge between $v_{i}$ and $v_{j}$ if and only if $v_{i} \cdot v_{j}=1$ for $i, j=1, \ldots, n$. The number of connected components of the intersection graph of $S$ will be denoted by $c(S)$.

We shall say that an element $v_{j} \in S$ is isolated, final or internal if the quantity

$$
\sum_{i=1, i \neq j}^{n}\left(v_{i} \cdot v_{j}\right)
$$

is equal to, respectively, 0,1 or 2 . In other words, $v_{j}$ is isolated or final if it is, respectively, an isolated vertex or a leaf of the intersection graph, and it is internal otherwise.

Given elements $e, v \in \mathbf{D}^{n}$ with $e \cdot e=-1$, we shall denote by $\pi_{e}(v)$ the projection of $v$ in the direction orthogonal to $e$ :

$$
\pi_{e}(v):=v+(v \cdot e) e \in \mathbf{D}^{n} .
$$

Two elements $v, w \in \mathbf{D}^{n}$ are linked if there exists $e \in \mathbf{D}^{n}$ with $e \cdot e=-1$ such that

$$
v \cdot e \neq 0, \quad \text { and } \quad w \cdot e \neq 0 .
$$

A set $S \subseteq \mathbf{D}^{n}$ is irreducible if, given two elements $v, w \in S$, there exists a finite sequence

$$
v_{0}=v, v_{1}, \ldots, v_{k}=w \in S
$$


such that $v_{i}$ and $v_{i+1}$ are linked for $i=0, \ldots, k-1$. A set which is not irreducible is reducible.

The reason to introduce the following definition is technical. It will become clear later on (see the "Outline" at the beginning of Section 3).

Definition 2.2 A subset $S=\left\{v_{1}, \ldots, v_{n}\right\} \subseteq \mathbf{D}^{n}$ is good if it is irreducible and its elements satisfy $(2-1)$.

Definition 2.3 Given a subset $S=\left\{v_{1} \ldots, v_{n}\right\} \subseteq \mathbf{D}^{n}$, let

$$
I(S):=\sum_{i=1}^{n}\left(-v_{i} \cdot v_{i}-3\right) \in \mathbb{Z} .
$$

The following Lemma will be used in Section 3, Section 5, Section 6 and Section 8.

Lemma 2.4 Let $S=\left\{v_{1}, v_{2}, v_{3}\right\} \subseteq \mathbf{D}^{3}=\left\langle e_{1}, e_{2}, e_{3}\right\rangle$ be a good subset with $I(S)<0$. Then, up to applying to $S$ an element of $\operatorname{Aut}\left(\mathbf{D}^{3}\right)$ and possibly replacing $\left(v_{1}, v_{2}, v_{3}\right)$ with $\left(v_{3}, v_{2}, v_{1}\right)$, one of the following holds:

(1) $\left(v_{1}, v_{2}, v_{3}\right)=\left(e_{1}-e_{2}, e_{2}-e_{3},-e_{2}-e_{1}\right)$,

(2) $\left(v_{1}, v_{2}, v_{3}\right)=\left(e_{1}-e_{2}, e_{2}-e_{3}, e_{1}+e_{2}+e_{3}\right)$ and

(3) $\left(v_{1}, v_{2}, v_{3}\right)=\left(e_{1}+e_{2}+e_{3},-e_{1}-e_{2}+e_{3}, e_{1}-e_{2}\right)$.

Moreover,

$$
\left(p_{1}(S), p_{2}(S), c(S), I(S)\right)= \begin{cases}(1,1,1,-3) & \text { in case (1) } \\ (0,2,2,-2) & \text { in case (2) } \\ (0,1,2,-1) & \text { in case (3). }\end{cases}
$$

In particular, $\left(a_{1}, a_{2}, a_{3}\right) \in\{(2,2,2),(2,2,3),(3,3,2)\}$.

Proof Up to replacing $\left(v_{1}, v_{2}, v_{3}\right)$ with $\left(v_{3}, v_{2}, v_{1}\right)$, by Conditions (2-1) we have three possible cases: (a) $v_{1} \cdot v_{2}=v_{2} \cdot v_{3}=1$, (b) $v_{1} \cdot v_{2}=1, v_{2} \cdot v_{3}=0$ and (c) $v_{1} \cdot v_{2}=v_{2} \cdot v_{3}=0$. Moreover, since $I(S)<0$ we have $\sum_{i} a_{i} \leq 8$. Therefore $a_{i} \leq 4$ for $i=1,2,3$. Using the fact that $S$ is irreducible it is easy to see that $a_{i}<4$ for $i=1,2,3$. This implies

$$
\left\{a_{1}, a_{2}, a_{3}\right\} \in\{\{2,2,2\},\{2,2,3\},\{3,3,2\}\} .
$$

Now observe that if $a_{i}=3$ then, up to applying an element of $\operatorname{Aut}\left(\mathbf{D}^{n}\right)$ we have $v_{i}=e_{1}+e_{2}+e_{3}$. If $a_{j}=2$ then $v_{j} \in\left\{ \pm e_{l} \pm e_{m}\right\}$, therefore $v_{i} \cdot v_{j}$ is an even number, 
hence $v_{i} \cdot v_{j}=0$. By a similar argument one sees that there cannot be distinct elements $v_{i}$ and $v_{j}$ with $a_{i}=a_{j}=3$ and $v_{i} \cdot v_{j}=0$. Using such considerations it is easy to check that, up to replacing $\left(v_{1}, v_{2}, v_{3}\right)$ with $\left(v_{3}, v_{2}, v_{1}\right)$,

(a) $\left(a_{1}, a_{2}, a_{3}\right)=(2,2,2)$ is the only triple compatible with case (a),

(b) $\left(a_{1}, a_{2}, a_{3}\right)=(2,2,3)$ is the only triple compatible with case (b) and

(c) $\left(a_{1}, a_{2}, a_{3}\right)=(3,3,2)$ is the only triple compatible with case (c).

The lemma follows by a straightforward case-by-case analysis.

The following lemma provides a basic constraint on $p_{1}(S)$ and $p_{2}(S)$ coming from the assumption $I(S)<0$. It will be used in Section 4 and Section 5.

Lemma 2.5 Let $S \subseteq \mathbf{D}^{n}=\left\langle e_{1}, \ldots, e_{n}\right\rangle$ be a subset of cardinality $n$ with $I(S)<0$. Then,

$$
2 p_{1}(S)+p_{2}(S)>\sum_{j=4}^{n}(j-3) p_{j}(S)
$$

Proof Let $S=\left\{v_{1}, \ldots, v_{n}\right\}$ and let $M=\left(m_{i j}\right)$ be the matrix defined by $v_{i}=$ $\sum_{j} m_{i j} e_{j}$. By the definition of $p_{i}(S)$, the number of non-zero entries of $M$ is

$$
\sum_{i=1}^{n} i p_{i}(S) \leq \sum_{i, j}\left|m_{i j}\right| \leq \sum_{i, j} m_{i j}^{2}=-\sum_{i=1}^{n} v_{i} \cdot v_{i}
$$

Moreover, the assumption $I(S)<0$ is equivalent to

$$
-\sum_{i=1}^{n} v_{i} \cdot v_{i}<3 n
$$

Since it is also evident that

$$
n=p_{1}(S)+p_{2}(S)+\cdots+p_{n}(S),
$$

the lemma follows.

Given integers $a_{1}, \ldots, a_{n} \geq 2$, we shall use the notation

$$
\left[a_{1}, \ldots, a_{n}\right]^{-}:=a_{1}-\frac{1}{a_{2}-\frac{1}{\ddots-\frac{1}{a_{n}}}},
$$


and for any integer $t \geq 0$ we shall write

$$
\left(\ldots, 2^{[t]}, \ldots\right):=(\ldots, \overbrace{2, \ldots, 2}^{t}, \ldots) .
$$

The following arithmetic lemma will be used in the last section of the paper to prove Theorem 1.2.

Lemma 2.6 Let $p>q \geq 1$ be coprime integers, and suppose that

$$
\frac{p}{q}=\left[a_{1}, \ldots, a_{n}\right]^{-}, \quad \frac{p}{p-q}=\left[b_{1}, \ldots, b_{m}\right]^{-},
$$

with $a_{1}, \ldots, a_{n} \geq 2$ and $b_{1}, \ldots, b_{m} \geq 2$. Then,

$$
\sum_{i=1}^{n}\left(a_{i}-3\right)+\sum_{j=1}^{m}\left(b_{j}-3\right)=-2 .
$$

Proof We can write

$$
\frac{p}{q}=\left[m_{1}, 2^{\left[m_{2}\right]}, m_{3}, 2^{\left[m_{4}\right]}, \ldots, m_{2 s-1}, 2^{\left[m_{2 s}\right]}\right]^{-}
$$

for some

$$
m_{1}, m_{3}, \ldots, m_{2 s-1} \geq 3, \quad m_{2}, m_{4}, \ldots, m_{2 s} \geq 0 .
$$

Then, by Riemenschneider's point rule [10]

$$
\frac{p}{p-q}=\left[2^{\left[m_{1}-2\right]}, m_{2}+3,2^{\left[m_{3}-3\right]}, m_{4}+3, \ldots, 2^{\left[m_{2 s-1}-3\right]}, m_{2 s}+2\right]^{-} .
$$

Therefore,

$$
\sum_{i=1}^{n}\left(a_{i}-3\right)=\sum_{i=1}^{s}\left(m_{2 i-1}-3\right)-\sum_{i=1}^{s} m_{2 i}
$$

and

$$
\sum_{j=1}^{m}\left(b_{j}-3\right)=-1+\sum_{i=1}^{s} m_{2 i}-\sum_{i=1}^{s}\left(m_{2 i-1}-3\right)-1 .
$$

The lemma follows immediately.

\section{The case $p_{1}(S)>0$ and $I(S)<0$}

Outline In this section we introduce the key notion of standard subset, which is the algebraic object naturally arising in our approach to Theorem 1.2 (see the outline of the proof in Section 1). For technical reasons, in order to understand standard subsets we need to understand first the more general good subsets introduced in Section 2. In this section we study the special class of good subsets $S$ satisfying $p_{1}(S)>0$ and 
$I(S)<0$. As explained at the beginning of Section 4, this is one of the two important classes of good subsets $S$ with $I(S)<0$. The main result of this section is Corollary 3.5 , which shows that a good subset with $p_{1}(S)>0$ and $I(S)<0$ is necessarily standard and is obtained from a standard subset of $\mathbf{D}^{3}$ by a finite sequence of operations we call expansions (see Definition 3.4). The results of this section will be used in Section 5 .

Definition 3.1 A subset $S_{n}=\left\{v_{1}, \ldots, v_{n}\right\} \subseteq \mathbf{D}^{n}$ such that

$$
v_{i} \cdot v_{j}= \begin{cases}-a_{i} \leq-2 & \text { if } i=j, \\ 1 & \text { if }|i-j|=1, \\ 0 & \text { if }|i-j|>1 .\end{cases}
$$

for $i, j=1, \ldots, n$ will be called standard.

The following lemma deals with good subsets $S$ satisfying $p_{1}(S)>0$. It will be used in the proofs of Proposition 3.3, Corollary 3.5 and in Section 4.

Lemma 3.2 Suppose that $n>3$, and let

$$
S_{n}=\left\{v_{1}, \ldots, v_{n}\right\} \subseteq \mathbf{D}^{n}=\left\langle e_{1}, \ldots, e_{n}\right\rangle
$$

be a good subset such that $E_{i}^{S_{n}}=\{s\}$ for some $i, s \in\{1, \ldots, n\}$. Then,

(1) $v_{s}$ is internal;

(2) for some $1 \leq j \leq n$ we have $V_{s}=\{i, j\}, E_{j}^{S_{n}}=\{s-1, s, s+1\}$ and $\left|v_{s-1} \cdot e_{j}\right|=$ $\left|v_{s} \cdot e_{j}\right|=\left|v_{s+1} \cdot e_{j}\right|=1$ and

(3) for some $t \in\{s-1, s+1\}$ the set

$$
S_{n-1}:=S_{n} \backslash\left\{v_{s}, v_{t}\right\} \cup\left\{\pi_{e_{j}}\left(v_{t}\right)\right\} \subseteq\left\langle e_{1}, \ldots, e_{i-1}, e_{i+1}, \ldots, e_{n}\right\rangle \cong \mathbf{D}^{n-1}
$$

is $\operatorname{good},\left|E_{j}^{S_{n-1}}\right|=1$ and $I\left(S_{n-1}\right)=I\left(S_{n}\right)+2+v_{s} \cdot v_{s}$.

Moreover, if $S_{n}$ is standard then so is $S_{n-1}$.

Proof Since $S_{n}$ is irreducible we have $\left|V_{s}\right| \geq 2$. If $\left|V_{s}\right|>2$, the set obtained from $S_{n}$ by replacing $v_{s}$ with $\pi_{e_{i}}\left(v_{s}\right)$ would still satisfy (2-1), but it would consist of $n$ independent vectors (see Remark 2.1) contained in the span of the $n-1$ vectors $e_{1}, \ldots, e_{i-1}, e_{i+1}, \ldots, e_{n}$, giving a contradiction. Therefore $\left|V_{s}\right|=2$, ie $V_{s}=\{i, j\}$ for some $j \neq i$. 
If $\left|v_{s} \cdot e_{j}\right|>1$ then we get a contradiction as before by replacing $v_{s}$ with $\pi_{e_{i}}\left(v_{s}\right)$. Hence, we conclude $\left|v_{s} \cdot e_{j}\right|=1$. Since $S_{n}$ is irreducible and $E_{i}^{S_{n}}=\{s\}, v_{s}$ is not isolated.

We need to show that $v_{s}$ is not final. By contradiction, suppose eg that $v_{s-1} \cdot v_{s}=0$ and $v_{s} \cdot v_{s+1}=1$ (the discussion in the case $v_{s-1} \cdot v_{s}=1, v_{s} \cdot v_{s+1}=0$ is similar). Let $l \geq 1$ be the largest natural number such that the set $\left\{v_{s}, \ldots, v_{s+l}\right\}$ has connected intersection graph. If

$$
a_{s+1}=\cdots=a_{s+l}=2
$$

it is easy to check that $\left|\cup_{i=0}^{l} V_{s+i}\right|=l+2$. Since $S_{n}$ is irreducible and $E_{i}^{S_{n}}=\{s\}$, this gives a contradiction. Therefore $a_{s+h}>2$ for some $1 \leq h \leq l$. Choose $h$ to be as small as possible. Then, it is easy to verify that for some $k \in\{1, \ldots, n\}$

$$
V_{s+h} \cap V_{s+h-1}=\left\{e_{k}\right\} \text { and }\left|v_{s+h} \cdot e_{k}\right|=1 .
$$

Since $\left|\cup_{i=0}^{h-1} V_{s+i}\right|=h+1$, it follows that by eliminating the vectors

$$
v_{s}, v_{s+1}, \ldots, v_{s+h-1}
$$

and replacing $v_{s+h}$ with $\pi_{e_{k}}\left(v_{s+h}\right)$ one obtains a set of $n-h$ independent vectors contained in the span of $n-(h+1)$ vectors. This contradiction shows that $v_{s}$ must be internal, ie $v_{s-1} \cdot v_{s}=v_{s+1} \cdot v_{s}=1$.

Now observe that, since $E_{i}^{S_{n}}=\{s\}$, we must have $j \in V_{s-1} \cap V_{s+1}$. If $a_{s-1}=a_{s+1}=2$ then $v_{s-1} \cdot v_{s+1}=0$ implies $V_{s-1}=V_{s+1}$, and it is easy to verify that either $n=3$ or $S$ is reducible.

If $a_{s-1}, a_{s+1}>2$ then, since clearly $\left|v_{s-1} \cdot e_{j}\right|=\left|v_{s+1} \cdot e_{j}\right|=1$, one gets a contradiction by eliminating $v_{s}$ and replacing $v_{s-1}$ and $v_{s+1}$, respectively, with $\pi_{e_{j}}\left(v_{s-1}\right)$ and $\pi_{e_{j}}\left(v_{s+1}\right)$. We conclude that either (i) $a_{s-1}>2$ and $a_{s+1}=2$ or (ii) $a_{s+1}>2$ and $a_{s-1}=2$. By symmetry, it suffices to consider the case $a_{s+1}>2$ and $a_{s-1}=2$. Since $\left|v_{s-1} \cdot e_{j}\right|=\left|v_{s+1} \cdot e_{j}\right|=1$, we have $v_{s-1} \cdot \pi_{e_{j}}\left(v_{s+1}\right)=1$. Therefore the elements of the set

$$
S_{n-1}:=\left\{v_{1}, \ldots, v_{n}\right\} \backslash\left\{v_{s}, v_{s+1}\right\} \cup\left\{\pi_{e_{j}}\left(v_{s+1}\right)\right\}
$$

satisfy (2-1). Moreover, the formula

$$
I\left(S_{n-1}\right)=I\left(S_{n}\right)+2+v_{s} \cdot v_{s}
$$

is straightforward to check. Since $E_{i}^{S_{n}}=\{s\}$ we have $E_{j}^{S_{n}}=\{s-1, s, s+1\}$, therefore the only vectors linked to $v_{s}$ are $v_{s-1}$ and $v_{s+1}$. Since $v_{s-1}$ and $\pi_{e_{j}}\left(v_{s+1}\right)$ are linked to each other, it follows easily that $S_{n-1}$ is irreducible. The fact that if $S_{n}$ is standard then so is $S_{n-1}$ is evident from the definition of $S_{n-1}$. 
The following proposition analyzes the nature of a good subset $S$ with $I(S)<0$ and $p_{1}(S)>0$. It is essential to prove the main result of this section, ie Corollary 3.5.

Proposition 3.3 Suppose that $n \geq 3$, and let

$$
S=\left\{v_{1}, \ldots, v_{n}\right\} \subseteq \mathbf{D}^{n}=\left\langle e_{1}, \ldots, e_{n}\right\rangle
$$

be a good subset such that $I(S)<0$ and $p_{1}(S)>0$. Then,

(1) $S$ is standard;

(2) $\left|v_{i} \cdot e_{j}\right| \leq 1$ for every $i, j=1, \ldots, n$ and

(3) If $n \geq 4$ there exist $h, t \in\{1, \ldots, n\}$ and $s \in\{1, n\}$ such that

$$
E_{h}^{S}=\{s, t\}, a_{s}=2 \text { and } a_{t}>2 .
$$

Proof If $n=3$ the proposition follows from Lemma 2.4. If $n>3$, set $S_{n}:=S$. By Lemma 3.2 there exists a good subset $S_{n-1}$ with $p_{1}\left(S_{n-1}\right)>0$, to which Lemma 3.2 can be applied again as long as $n-1>3$. Applying the lemma $n-3$ times we obtain a sequence $S_{n}, S_{n-1}, \ldots, S_{3}$ of good subsets with $p_{1}\left(S_{n}\right), \ldots, p_{1}\left(S_{3}\right)>0$. In particular, the fact that $S_{3}$ is good and $p_{1}\left(S_{3}\right)>0$ implies, by Lemma 2.4, that there is only one possibility for $S_{3}$ modulo the action of $\operatorname{Aut}\left(\mathbf{D}^{3}\right)$, which is given by Lemma 2.4(1). This immediately implies that all the sets $S_{i}, i=3, \ldots, n$, have connected intersection graph. Therefore $S_{n}$ is standard, ie (1) holds. Since by assumption $I\left(S_{n}\right) \leq-1$ and $I\left(S_{3}\right)=-3$, the formula for $I\left(S_{n-1}\right)$ in the statement of Lemma 3.2 implies that every time we applied the lemma we had $v_{s} \cdot v_{s} \geq-4$. Since $V_{s}=\{i, j\}$, this implies $v_{s} \cdot v_{s}=-2$. Therefore $\left|v_{s} \cdot e_{i}\right|=\left|v_{s} \cdot e_{j}\right|=1$, and by the definition of the sequence $S_{n}, S_{n-1}, \ldots, S_{3}$ this immediately implies (3). Finally, it is easy to check that (2) holds for $S_{3}$ and $S_{4}$, and that if $S_{k-1}$ is obtained from $S_{k}$ as in Lemma 3.2 and (2) holds for $S_{k-1}$ then (2) holds for $S_{k}$. This proves (2) and concludes the proof.

Definition 3.4 Let $S=\left\{v_{1}, \ldots, v_{n}\right\} \subseteq \mathbf{D}^{n}$ be a subset satisfying (2-1) and such that $\left|v_{i} \cdot e_{j}\right| \leq 1$ for every $i, j=1, \ldots, n$. Suppose that there exist $1 \leq h, s, t \leq n$ such that

$$
E_{h}^{S}=\{s, t\} \text { and } a_{t}>2 .
$$

Then, we say that the subset $S^{\prime} \subseteq\left\langle e_{1}, \ldots, e_{h-1}, e_{h+1}, \ldots, e_{n}\right\rangle \cong \mathbf{D}^{n-1}$ defined by

$$
S^{\prime}:=S \backslash\left\{v_{s}, v_{t}\right\} \cup\left\{\pi_{e_{h}}\left(v_{t}\right)\right\}
$$

is obtained from $S$ by a contraction, and we write $S \searrow S^{\prime}$. Moreover, we say that $S$ is obtained from $S^{\prime}$ by an expansion, and we write $S^{\prime} \nearrow S$. 
The following result will be used in the proof of Corollary 5.4.

Corollary 3.5 Suppose that $n \geq 3$, and let $S=\left\{v_{1}, \ldots, v_{n}\right\} \subseteq \mathbf{D}^{n}$ be a good subset such that $I(S)<0$ and $p_{1}(S)>0$. Then, $S$ is standard and there is a sequence of expansions

$$
S_{3} \nearrow S_{4} \nearrow \cdots \nearrow S_{n-1} \nearrow S_{n}:=S
$$

such that $S_{k}$ is standard and $I\left(S_{k}\right)=-3$ for every $k=3, \ldots, n$.

Proof If $n=3$ the corollary follows from Lemma 2.4. Suppose that $n \geq 4$, let $S_{n}:=S$, and let $h, s$ and $t$ be the indexes appearing in Proposition 3.3(3). By Lemma 3.2 and Proposition 3.3, the set

$$
S_{n-1}:=S_{n} \backslash\left\{v_{s}, v_{t}\right\} \cup\left\{\pi_{e_{h}}\left(v_{t}\right)\right\}
$$

is standard and is obtained from $S_{n}$ by a contraction. Moreover, $p_{1}\left(S_{n-1}\right)>0$, and since $a_{s}=2$ we have $I\left(S_{n-1}\right)=I\left(S_{n}\right)$. Arguing in the same way we get a sequence of contractions $S_{n} \searrow S_{n-1} \searrow \cdots \searrow S_{3}$ with each $S_{k}$ standard and $I\left(S_{k}\right)=I\left(S_{3}\right)$ for every $k$. Since by Lemma 2.4 we have $I\left(S_{3}\right)=-3$, this concludes the proof.

\section{The case $p_{1}(S)=0, p_{2}(S)>0$ and $I(S)<0$}

Outline It follows from Lemma 2.5 that if a subset $S \subset \mathbf{D}^{n}$ of cardinality $n$ satisfies $I(S)<0$ and $p_{1}(S)=0$, then necessarily $p_{2}(S)>0$. Having dealt with the case $p_{1}(S)>0$ in the previous section, in this section we start tackling the more difficult case of a good subset with $p_{1}(S)=0, p_{2}(S)>0$ and $I(S)<0$. As in the previous case, one would like to show that good subsets satisfying this condition are obtained by expansions of smaller subsets of the same type. But in this case one must first understand the potential obstruction coming from the fact that during a sequence of contractions the subset might develop what we call bad components (see Definition 4.1). The main result of the section is Proposition 4.5, essentially giving a control on the number of bad components which might appear as a result of contractions. In the next section we shall use Proposition 4.5 to establish some results which hold under the general assumption $I(S)<0$ and, using these, in Section 6 we shall finally be able to show that any standard subset $S$ with $I(S)<0$ is obtained by expanding standard subsets of the same type.

Definition 4.1 Let $S^{\prime}=\left\{v_{1}, \ldots, v_{n}\right\} \subseteq \mathbf{D}^{n}, n \geq 3$, be a good subset, and suppose there exists $1<s<n$ such that $C^{\prime}=\left\{v_{s-1}, v_{s}, v_{s+1}\right\} \subseteq S^{\prime}$ gives a connected component of the intersection graph of $S^{\prime}$, with $v_{s-1} \cdot v_{s-1}=v_{s+1} \cdot v_{s+1}=-2, v_{s} \cdot v_{s}<-2$ 
and $E_{j}^{S^{\prime}}=\{s-1, s, s+1\}$ for some $j$. Let $S \subseteq \mathbf{D}^{m}$ be a subset of order $m \geq n$ obtained from $S^{\prime}$ by a sequence of expansions by final (-2)-vectors attached to $C^{\prime}$, so that $c(S)=c\left(S^{\prime}\right)$ and there is a natural $1-1$ correspondence between the sets of connected components of the intersection graphs of $S$ and $S^{\prime}$. Then, the connected component $C \subseteq S$ corresponding to $C^{\prime} \subseteq S^{\prime}$ is a bad component of $S$. The number of bad components of $S$ will be denoted by $b(S)$.

If a good subset $S=\left\{v_{1}, \ldots, v_{n}\right\} \subseteq \mathbf{D}^{n}$ satisfies $p_{2}(S)>0$ then for some $i, s, t \in$ $\{1, \ldots, n\}$ we must have $E_{i}^{S}=\{s, t\}$. There are two possibilities: either $a_{s}$ and $a_{t}$ are both greater then 2 , or one of them is equal to 2 . The next lemma analyzes with the latter possibility (assuming $S$ has no bad components), while the former possibility is considered in Lemma 4.3.

Lemma 4.2 Suppose that $n>3$, the subset

$$
S=\left\{v_{1}, \ldots, v_{n}\right\} \subseteq \mathbf{D}^{n}=\left\langle e_{1}, \ldots, e_{n}\right\rangle
$$

is good, has no bad components and there exist $i, s, t \in\{1, \ldots, n\}$ such that

$$
E_{i}^{S}=\{s, t\} \text { and } a_{s}=2 .
$$

Then, one of the following holds.

(1) $v_{s} \cdot v_{t}=0, v_{s}$ is not internal, $\left|V_{t}\right|>2$, and the set

$$
S^{\prime}:=S \backslash\left\{v_{s}, v_{t}\right\} \cup\left\{\pi_{e_{i}}\left(v_{t}\right)\right\} \subseteq\left\langle e_{1}, \ldots, e_{i-1}, e_{i+1} \ldots, e_{n}\right\rangle \cong \mathbf{D}^{n-1}
$$

is good. Moreover, $I\left(S^{\prime}\right) \leq I(S)$ and $S^{\prime}$ has no bad components.

(2) $v_{s} \cdot v_{t}=0, v_{s}$ is internal and $a_{t}>2$.

(3) $v_{s} \cdot v_{t}=1, a_{t}>2$ and the set $S^{\prime}$ defined in (1) above is good. Moreover, $I\left(S^{\prime}\right) \leq I(S)$ and $S^{\prime}$ has no bad components.

Proof Since $a_{s}=2$, we have $V_{s}=\{i, j\}$ for some $i, j \in\{1, \ldots, n\}$.

First case $\left(v_{s} \cdot v_{t}=0\right.$ and $\left.a_{t}=2\right) \quad$ In this case $V_{t}=\{i, j\}$. Since $S$ is irreducible and $n>3$, there is a $v_{r}$ with $r \notin\{s, t\}$ linked to either $v_{s}$ or $v_{t}$. Since $E_{i}^{S}=\{s, t\}$, this implies $j \in V_{r}$ and $v_{r} \cdot v_{S}=v_{r} \cdot v_{t}=1$, therefore $\left|V_{r}\right| \geq 2$. Assuming $\left|V_{r}\right|=2$ it easily follows that $S$ is reducible. Therefore $\left|V_{r}\right|>2$, which implies that $S$ has a bad component. Hence this case cannot occur.

Second case $\left(v_{s} \cdot v_{t}=0\right.$ and $\left.a_{t}>2\right)$ We have $V_{t} \supseteq\{i, j\}$. Suppose first that $v_{s}$ is isolated. If $\left|V_{t}\right|=2$, then no other vector could link $v_{s}$ nor $v_{t}$, and $S$ would be reducible. If $\left|V_{t}\right|>2$ then the set

$$
S^{\prime}:=S \backslash\left\{v_{s}, v_{t}\right\} \cup\left\{\pi_{e_{i}}\left(v_{t}\right)\right\}
$$


satisfies (2-1). Since every vector $v$ linked to $v_{s}$ must satisfy $v \cdot e_{j} \neq 0, S^{\prime}$ is irreducible. Clearly $I\left(S^{\prime}\right) \leq I(S)$ and it is easy to check that $b\left(S^{\prime}\right)=b(S)=0$. Hence (1) holds. If $v_{s}$ is final then $v_{s} \cdot v_{s^{\prime}}=1$ for some $s^{\prime} \in\{s-1, s+1\}$. This implies $\left|v_{s^{\prime}} \cdot e_{j}\right|=1$. If $\left|V_{t}\right|>2$ then it follows as above that the set $S^{\prime}$ is good, $I\left(S^{\prime}\right) \leq I(S)$, and $b\left(S^{\prime}\right)=b(S)=0$. Hence (1) holds. If $\left|V_{t}\right|=2$ then $v_{s^{\prime}} \cdot v_{t}=1$, hence $\left|v_{t} \cdot e_{j}\right|=1$. Since $v_{s} \cdot v_{t}=0$, we also have $\left|v_{t} \cdot e_{i}\right|=1$. But this is impossible because $a_{t}>2$.

Third case $\left(v_{s} \cdot v_{t}=1\right.$ and $\left.a_{t}=2\right) \quad$ In this case $v_{s}$ is not isolated and $V_{t}=\{i, k\}$ for some $k \neq j$. Observe that $v_{s}$ cannot be a final vector, otherwise $E_{j}^{S}=\{s\}$, which by Lemma 3.2 implies that $v_{s}$ is internal. By symmetry, we may assume without loss of generality that $t=s+1$. Then, arguing as in the proof of Lemma 3.2, one gets a contradiction using the fact that $E_{i}^{S}=\{s, t\}$ by considering the largest $l, m \geq 1$ such that $\left\{v_{s-m}, \ldots, v_{s+l}\right\}$ has connected intersection graph. In fact, if

$$
a_{s-m}=\cdots=a_{s+l}=2
$$

it is easy to check that $\left|\cup_{i=s-m}^{s+l} V_{i}\right|=l+m+2$. Since $S$ is irreducible and $E_{i}^{S}=$ $\{s, s+1\}$, this easily leads to a contradiction. Therefore, $a_{r}>2$ for some $s-m \leq r<s$ or $a_{p}>2$ for some $s+1<p \leq s+l$. Suppose eg that only the latter happens (the other cases can be dealt with similarly). Choose $p$ as small as possible. Then, for some $q \in\{1, \ldots, n\}$

$$
V_{p} \cap V_{p-1}=\left\{e_{q}\right\} \text { and }\left|v_{p} \cdot e_{q}\right|=1 .
$$

Since $\left|\cup_{i=s-m}^{p-l} V_{i}\right|=p+1-s+m$, one obtains the contradiction by eliminating the vectors

$$
v_{s-m}, \ldots, v_{p-1},
$$

replacing $v_{p}$ with $\pi_{e_{q}}\left(v_{p}\right)$ and arguing as in the proof of Lemma 3.2.

Fourth case $\left(v_{s} \cdot v_{t}=1\right.$ and $\left.a_{t}>2\right) \quad$ By symmetry we may assume $t=s+1$. If $j \notin V_{s+1}$ then, as in the case $a_{t}=2, v_{s}$ is not final, otherwise $E_{j}^{S}=\{s\}$, which implies that $v_{s}$ is internal by Lemma 3.2. Then one obtains a contradiction as in the previous case by considering the biggest $l \geq 0$ such that $\left\{v_{s-l}, \ldots, v_{s}\right\}$ has connected intersection graph.

If $V_{s+1}=\{i, j\}$ then $v_{s}$ cannot be final because otherwise $v_{s}$ and $v_{s+1}$ would be linked to no other vector, and therefore the set $S$ would be reducible. But if $v_{s}$ is not final then $E_{j}^{S} \supseteq\{s-1, s, s+1\}$ and $k \in V_{s-1} \cap V_{s+1}$ for some $k \notin\{i, j\}$, which is impossible if $V_{s+1}=\{i, j\}$.

Therefore we conclude that $V_{s+1} \supsetneqq\{i, j\}$. Since if $v_{h}$ is linked to $v_{s}$ then $j \in V_{h}$, it follows that the set

$$
S^{\prime}=S \backslash\left\{v_{s}, v_{s+1}\right\} \cup\left\{\pi_{e_{i}}\left(v_{s+1}\right)\right\}
$$


is good, and it is clear that $I\left(S^{\prime}\right) \leq I(S)$. Moreover, one can easily check that $b\left(S^{\prime}\right)=b(S)=0$. Hence (3) holds.

Lemma 4.3 Suppose that $n>3$, the subset

$$
S=\left\{v_{1}, \ldots, v_{n}\right\} \subseteq \mathbf{D}^{n}=\left\langle e_{1}, \ldots, e_{n}\right\rangle
$$

is good, has no bad components and there exist $i, s, t \in\{1, \ldots, n\}$ such that

$$
E_{i}^{S}=\{s, t\} \text { and } a_{s}, a_{t}>2 .
$$

Then, up to replacing the pair $(s, t)$ with another pair satisfying (4-1), one of the following holds.

(1) The set

$$
S^{\prime}=S \backslash\left\{v_{s}, v_{t}\right\} \cup\left\{\pi_{e_{i}}\left(v_{t}\right)\right\} \subseteq\left\langle e_{1}, \ldots, e_{i-1}, e_{i+1}, \ldots, e_{n}\right\rangle \cong \mathbf{D}^{n-1}
$$

is good, $I\left(S^{\prime}\right) \leq I(S)-1$ and $b\left(S^{\prime}\right) \leq 1$.

(2) There exist $k \neq i$ and $s^{\prime} \in\{s-1, s+1\}$ such that

(a) $E_{k}^{S}=\left\{s, s^{\prime}\right\}$

(b) $v_{s^{\prime}} \cdot v_{s}=1$ and

(c) $a_{s^{\prime}}=2$.

Proof If the set $S^{\prime}$ of (1) is good, since $a_{s}>2$ it follows that $I\left(S^{\prime}\right) \leq I(S)-1$, and it is easy to check that $S^{\prime}$ can have at most one bad component, therefore $b\left(S^{\prime}\right) \leq 1$. Hence (1) holds.

Now suppose that the set

$$
S^{\prime}(s, t):=S \backslash\left\{v_{s}, v_{t}\right\} \cup\left\{\pi_{e_{i}}\left(v_{t}\right)\right\}
$$

is not good because $\pi_{e_{i}}\left(v_{t}\right)$ happens to have square -1 . Then, $v_{t}=x e_{i} \pm e_{j}$ with $|x|>1$. Since $v_{s} \cdot v_{t} \in\{0,1\}$, we must have $j \in V_{s}$. Moreover, the vector $\pi_{e_{i}}\left(v_{s}\right)$ must have square less than -1 , because otherwise $v_{s}=y e_{i} \pm e_{j}$ with $|y|>1$, which implies

$$
\left|v_{s} \cdot v_{t}\right|=|-x y \pm 1| \geq 3 \text {. }
$$

Therefore the set $S^{\prime}(t, s)$ satisfies $(2-1)$. Since there is no vector linked to $v_{t}$ but unlinked from $v_{s}$, it follows that $S^{\prime}(t, s)$ is irreducible as well. Therefore, after replacing $(s, t)$ with $(t, s)$, (1) holds.

We may now assume that $S^{\prime}(s, t)$ and $S^{\prime}(t, s)$ satisfy (2-1) but they are not good because they are both reducible. We can write $S^{\prime}(s, t)=S_{1}^{\prime} \cup S_{2}^{\prime}$, where $S_{2}^{\prime}$ is a maximal irreducible subset of $S^{\prime}(s, t)$ containing $\pi_{e_{i}}\left(v_{t}\right)$ and $S_{1}^{\prime}=S^{\prime}(s, t) \backslash S_{2}^{\prime}$. 
Define $S_{i} \subseteq S \backslash\left\{v_{s}\right\}, i=1,2$, to be the preimage of $S_{i}^{\prime}$ under the natural surjective map $S \backslash\left\{v_{s}\right\} \rightarrow S^{\prime}(s, t)$ sending $v_{j}$ to $v_{j}$ for $j \neq s, t$ and $v_{t}$ to $\pi_{e_{i}}\left(v_{t}\right)$. The decomposition $S \backslash\left\{v_{s}\right\}=S_{1} \cup S_{2}$ shows that $S \backslash\left\{v_{s}\right\}$ is reducible. Define

$$
V^{j}:=\cup_{v_{k} \in S_{j}} V_{k}, \quad j=1,2 .
$$

Observe that $V^{1} \cap V^{2}=\varnothing$ and $i \in V^{2}$, and let $V_{s}^{j}:=V_{s} \cap V^{j}, j=1,2$. Since $S$ is irreducible while $S \backslash\left\{v_{s}\right\}$ is reducible, there exists a vector $v_{r} \in S_{1}$ which is linked to $v_{s}$, therefore $\left|V_{s}^{1}\right| \geq 1$. If $\left|V_{s}^{1}\right|>1$ then we could replace $v_{s}$ with

$$
\tilde{v}_{s}:=-\sum_{k \in V_{s}^{1}}\left(v_{s} \cdot e_{k}\right) e_{k}
$$

and $v_{t}$ with $\pi_{e_{i}}\left(v_{t}\right)$. For every $u \neq s$ we would have either $V_{u} \cap V_{s} \subseteq V_{s}^{1}$ or $V_{u} \cap V_{s} \subseteq V_{s}^{2}$, implying that $v_{u} \cdot \widetilde{v}_{s} \in\{0,1\}$. The $n$ vectors resulting from the replacements just described would satisfy (2-1) and hence be independent, but they would be contained in the span of $\left\{e_{1}, \ldots, e_{n}\right\} \backslash\left\{e_{i}\right\}$, giving a contradiction. Thus, we have $V_{s}^{1}=\{k\}$ for some $k$. Since

$$
V_{s}^{1}=V_{s} \cap \bigcup_{v_{k} \in S_{1}} V_{k}=\bigcup_{v_{k} \in S_{1}}\left(V_{s} \cap V_{k}\right),
$$

if $v_{r} \in S_{1}$ is a vector linked to $v_{s}$ then $V_{r} \cap V_{s}=\{k\}$, hence $v_{r} \cdot v_{s}=1$. This implies that $\{s-1, s+1\} \cap E_{k}^{S} \neq \varnothing$. Moreover, if $v_{s}$ is final we have $r \in\{s-1, s+1\}$ and then $E_{k}^{S}=\{s-1, s\}$ or $E_{k}^{S}=\{s, s+1\}$. By symmetry, we may assume that the first case occurs. If $a_{s-1}=2$ then (2) holds. If $a_{s-1}>2$ and $k \notin V_{s+1}$, we can eliminate $v_{s}$, replace $v_{s-1}$ with $\pi_{e_{k}}\left(v_{s-1}\right)$ and $v_{t}$ with $\pi_{e_{i}}\left(v_{t}\right)$. This gives a contradiction unless $\pi_{e_{k}}\left(v_{s-1}\right)$ happens to have square -1 . But in that case we can replace $(s, t)$ with $(s-1, s)$, and by the argument given above $S^{\prime}(s-1, s)$ is good, therefore (1) holds.

Now we must consider the case when $v_{s}$ is not final. We have $v_{s-1} \cdot v_{s}=v_{s} \cdot v_{s+1}=1$, $E_{k}^{S}=\{s-1, s, s+1\}$ and $V_{s-1} \cap V_{s}=V_{s} \cap V_{s+1}=\{k\}$. Let us suppose that either $a_{s-1}>2$ or $a_{s+1}>2$. By symmetry we may assume that $a_{s-1}>2$. Since $v_{t} \notin S_{1}$, $t \notin\{s-1, s+1\}$, we have $v_{s} \cdot v_{t}=0$, so we can eliminate $v_{s}$ and make the replacements:

$$
v_{s-1} \mapsto \pi_{e_{k}}\left(v_{s-1}\right), \quad v_{t} \mapsto \pi_{e_{i}}\left(v_{t}\right) .
$$

It is easy to see that the resulting set $S^{\prime \prime}$ of $n-1$ vectors satisfies (2-1) and can be written as a disjoint union $S^{\prime \prime}=S_{1}^{\prime \prime} \cup \cdots \cup S_{q}^{\prime \prime}$ of maximal irreducible subsets so that each $S_{l}^{\prime \prime}$ is contained in the span of a set of vectors $e_{j}$ whose cardinality is equal to $\left|S_{l}^{\prime \prime}\right|$. We know that for some $l, v_{s+1} \in S_{l}^{\prime \prime}$. Moreover, by construction $E_{k}^{S_{l}^{\prime \prime}}=\{s+1\}$. Since $v_{s+1}$ is not internal in $S_{l}^{\prime \prime}$, we get a contradiction with Lemma 3.2(1). 
We are left with the case $a_{s-1}=a_{s+1}=2$. In this case it is easy to deduce that $V_{s-1}=V_{s+1}=\{h, k\}$ for some $h$, and $E_{h}^{S}=\{s-1, s+1\}$. But this means that $S$ contains a bad component, which is contrary to our assumptions.

The following is an auxiliary result which will be used in the proof of Proposition 4.5 as well as in Section 5.

\section{Lemma 4.4 Let}

$$
S=\left\{v_{1}, \ldots, v_{n}\right\} \subseteq \mathbf{D}^{n}=\left\langle e_{1}, \ldots, e_{n}\right\rangle
$$

be a good subset such that $p_{1}(S)=0, p_{2}(S)>0$ and $I(S)<0$. Suppose that for each $i, s, t \in\{1, \ldots, n\}$ such that $E_{i}^{S}=\{s, t\}$ we have either $a_{s}=2$ or $a_{t}=2$. Then, for at least one choice of $i, s, t$ such that $a_{s}=2$ and $E_{i}^{S}=\{s, t\}$, either $v_{s}$ is not internal or $v_{s} \cdot v_{t}=1$.

Proof Suppose by contradiction that for each $i, s, t$ such that $a_{s}=2$ and $E_{i}^{S}=\{s, t\}$ we have $v_{s} \cdot v_{t}=0$ and $v_{s}$ is internal. Then, if $V_{s}=\{i, j(i)\}$ it follows immediately that $j(i) \in V_{s-1} \cap V_{s} \cap V_{s+1}$, and therefore, since $v_{s} \cdot v_{t}=0$ implies $j(i) \in V_{t}$, we have $\left|E_{j(i)}^{S}\right| \geq 4$. Note that, in particular, we must have $n \geq 4$. Consider the collection $\{j(i)\}$ of all the indices $j(i)$ obtainable in this way. Since $p_{1}(S)=0$ and $\left|E_{j(i)}^{S}\right| \geq 4$ for every $i$, by Lemma 2.5 we have

$$
p_{2}(S)>p_{4}(S)+2 p_{5}(S)+\cdots+(n-3) p_{n}(S) \geq p_{4}(S)+p_{5}(S)+\cdots+p_{n}(S) .
$$

Therefore we must have $j(i)=j\left(i^{\prime}\right)$ for some $i \neq i^{\prime}$. But if $E_{i^{\prime}}^{S}=\left\{s^{\prime}, t^{\prime}\right\}$ with $a_{s^{\prime}}=2$ then $j(i)=j\left(i^{\prime}\right) \in V_{s^{\prime}-1} \cap V_{s^{\prime}} \cap V_{s^{\prime}+1}$ and $j(i)=j\left(i^{\prime}\right) \in V_{t^{\prime}}$, and this easily implies that $\left|E_{j(i)}^{S}\right| \geq 5$. Moreover, it is easy to check that if $i, i^{\prime}, i^{\prime \prime}$ are three distinct indexes with $\left|E_{i}^{S}\right|=\left|E_{i^{\prime}}^{S}\right|=\left|E_{i^{\prime \prime}}^{S}\right|=2$, then one cannot have $j(i)=j\left(i^{\prime}\right)=j\left(i^{\prime \prime}\right)$. This leads to conflict with Inequality (2-2), and we must conclude that there exist $i, s, t \in\{1, \ldots, n\}$ such that $a_{s}=2, E_{i}^{S}=\{s, t\}$ and either $v_{s}$ is not internal or $v_{s} \cdot v_{t}=1$.

Proposition 4.5 Suppose that $n>3$ and

$$
S=\left\{v_{1}, \ldots, v_{n}\right\} \subseteq \mathbf{D}^{n}=\left\langle e_{1}, \ldots, e_{n}\right\rangle
$$

is a good subset with no bad components and such that $p_{1}(S)=0, p_{2}(S)>0$ and $I(S)<0$. Then, there exist $i, s, t \in\{1, \ldots, n\}$ such that the set

$$
S^{\prime}=S \backslash\left\{v_{s}, v_{t}\right\} \cup\left\{\pi_{e_{i}}\left(v_{t}\right)\right\} \subseteq\left\langle e_{1}, \ldots, e_{i-1}, e_{i+1}, \ldots, e_{n}\right\rangle \cong \mathbf{D}^{n-1}
$$

is good. Moreover, $I\left(S^{\prime}\right) \leq I(S), b\left(S^{\prime}\right) \leq 1$ and if $b\left(S^{\prime}\right)=1$ then $v_{s} \cdot v_{s}<-2$ and $I\left(S^{\prime}\right) \leq I(S)-1$. 
Proof Since $p_{2}(S)>0$, there exist $i, s, t \in\{1, \ldots, n\}$ such that $E_{i}^{S}=\{s, t\}$. If $a_{s}>2$ and $a_{t}>2$ the hypotheses of Lemma 4.3 are satisfied. Therefore, since $S$ has no bad components, the conclusions of Lemma 4.3(1) or Lemma 4.3(2) hold.

If the conclusion of Lemma 4.3(1) holds, then the proposition follows immediately. If the conclusion of Lemma 4.3(2) holds then Lemma 4.2(3) applies and (2) holds. Therefore, from now on we assume that for each $i, s, t \in\{1, \ldots, n\}$ such that $E_{i}^{S}=\{s, t\}$ we have either $a_{s}=2$ or $a_{t}=2$. Since $p_{1}(S)=0, p_{2}(S)>0$ and $I(S)<0$, by Lemma 4.4, for at least one choice of $i, s, t$ we have $E_{i}^{S}=\{s, t\}, a_{s}=2$ and either $v_{s}$ is not internal or $v_{s} \cdot v_{t}=1$. Since we are assuming $b(S)=0$, by Lemma 4.2 we see that either the conclusion of Lemma 4.2(1) or the conclusion of Lemma 4.2(3) holds. In both cases the proposition is proved.

\section{The general case $I(S)<0$}

Outline In this section we study good subsets $S$ with no bad components and $I(S)<0$. Our aim is to establish some results which will be used in the next section to analyze standard subsets with $I(S)<0$. The main result is Corollary 5.4, which implies that a good subset $S$ with no bad components and $I(S)<0$ has $I(S) \in\{-1,-2,-3\}$ and is obtained by a sequence of expansions from a subset of $\mathbf{D}^{3}$ of the same kind as $S$.

The following simple lemma is used in the proof of Proposition 5.2.

Lemma 5.1 Let $S \subseteq \mathbf{D}^{n}, n \geq 3$, be a standard subset with $I(S)<0$. Then $S$ has no bad components.

Proof Since the intersection graph of a standard subset is connected, if the only connected component of the intersection graph of $S$ is bad, then by definition $S$ is obtained via expansions by final $(-2)$-vectors from a subset $S^{\prime} \subseteq \mathbf{D}^{3}$ consisting of a single bad component with $I\left(S^{\prime}\right)<0$. But Lemma 2.4 forbids the existence of such a subset.

The following proposition should be thought of as a generalization of Proposition $3.3(2)$.

Proposition 5.2 Suppose that $n \geq 3$, and let

$$
S=\left\{v_{1}, \ldots, v_{n}\right\} \subseteq \mathbf{D}^{n}=\left\langle e_{1}, \ldots, e_{n}\right\rangle
$$

be a good subset with no bad components such that $I(S)<0$. Then, $\left|v_{i} \cdot e_{j}\right| \leq 1$ for every $i, j=1, \ldots, n$. 
Proof We argue by induction on $n \geq 3$. If $n=3$ the conclusion is an immediate consequence of Lemma 2.4. Therefore, from now on we shall assume $n>3$. Since $I(S)<0$, by Lemma 2.5 Inequality (2-2) holds, therefore either $p_{1}(S)>0$ or $p_{2}(S)>0$. If $p_{1}(S)>0$ then the conclusion holds by Proposition 3.3, hence we may assume $p_{1}(S)=0$ and $p_{2}(S)>0$.

Since $b(S)=0$, by Proposition 4.5 there is a good subset $S^{\prime} \subseteq \mathbf{D}^{n-1}$ such that

$$
I\left(S^{\prime}\right)+b\left(S^{\prime}\right) \leq I(S)+b(S)<0 .
$$

In particular, it follows that $I\left(S^{\prime}\right)<0$. Now we set $S_{1}:=S, S_{2}:=S^{\prime}, n_{1}=n$ and $n_{2}=n-1$. If $n-1=3$ we stop. If $n-1 \geq 4$ and $p_{1}\left(S_{2}\right)>0$, then by Corollary $3.5 S_{2}$ is standard, $I\left(S_{2}\right)=-3$ and $S_{2}$ contracts to a standard subset $S_{3} \subseteq \mathbf{D}^{n-2}$ such that $I\left(S_{3}\right)=-3$. If $n-1 \geq 4$ and $p_{1}\left(S_{2}\right)=0$ then, since $I\left(S_{2}\right)<0$ we have $p_{2}\left(S_{2}\right)>0$. If $S_{2}$ has a bad component then there is a sequence of contractions from $S_{2}$ to a good subset $S_{3}^{\prime}$ with a connected component $\left\{v_{s-1}, v_{s}, v_{s+1}\right\}$ such that $v_{s-1} \cdot v_{s-1}=v_{s+1} \cdot v_{s+1}=-2, v_{s} \cdot v_{s}<-2$ and $E_{j}^{S_{3}^{\prime}}=\{s-1, s, s+1\}$ for some $j$. Then, we set

$$
S_{3}=S_{3}^{\prime} \backslash\left\{v_{s}, v_{s+1}\right\} \cup\left\{\pi_{e_{j}}\left(v_{s}\right)\right\} .
$$

It is easy to check that $S_{3}$ is good, has no bad components and $I\left(S_{3}\right)=I\left(S_{2}\right)+1$. Therefore, in any case we obtain a good subset $S_{3} \subseteq \mathbf{D}^{n_{3}}$ with $n_{3} \geq 2$ and

$$
I\left(S_{3}\right)+b\left(S_{3}\right) \leq I\left(S_{2}\right)+b\left(S_{2}\right)<0 .
$$

Continuing in this way, we obtain a decreasing sequence of good subsets without bad components

$$
S_{1} \subseteq \mathbf{D}^{n_{1}}, S_{2} \subseteq \mathbf{D}^{n_{2}}, \cdots, S_{k} \subseteq \mathbf{D}^{n_{k}}
$$

with $n_{1}>n_{2}>\cdots>n_{k} \geq 2$ and

$$
I\left(S_{i+1}\right)+b\left(S_{i+1}\right) \leq I\left(S_{i}\right)+b\left(S_{i}\right)<0, \quad i=1, \ldots, k-1 .
$$

Clearly a good subset $S \subseteq \mathbf{D}^{2}$ has $I(S)=-2$. Therefore, by Lemma 2.4 we have $I\left(S_{k}\right) \geq-3$ and $b\left(S_{k}\right)=0$. Setting

$$
\xi\left(S_{i}\right):= \begin{cases}I\left(S_{i}\right)+b\left(S_{i}\right) & i=1, \ldots, k \\ -3 & i=k+1\end{cases}
$$

since $b\left(S_{1}\right)=0$ we have

$$
\sum_{i=1}^{k}\left(\xi\left(S_{i}\right)-\xi\left(S_{i+1}\right)\right)=\xi\left(S_{1}\right)-\xi\left(S_{k+1}\right)=I\left(S_{1}\right)+3 \leq-1+3=2 .
$$


Since $\xi\left(S_{i}\right)-\xi\left(S_{i+1}\right) \geq 0$ for every $i$, we conclude

$$
0 \leq \xi\left(S_{i}\right)-\xi\left(S_{i+1}\right) \leq 2, \quad i=1, \ldots, k .
$$

In particular, $\xi\left(S_{1}\right)-\xi\left(S_{2}\right) \leq 2$. By a simple calculation one easily sees that this is equivalent to

$$
a_{s}+\left|v_{t} \cdot e_{i}\right|^{2} \leq b\left(S_{2}\right)+5 .
$$

First case $\left(b\left(S_{2}\right)=0\right) \quad$ In this case $a_{s} \geq 2$ implies $\left|v_{t} \cdot e_{i}\right|^{2}=1$ and therefore $a_{s} \leq 4$. Since $\left|V_{s}\right| \geq 2$, we necessarily have $\left|v_{s} \cdot e_{j}\right| \leq 1$ for every $j=1, \ldots, n$, and this easily implies the statement of the proposition.

Second case $\left(b\left(S_{2}\right)=1\right) \quad$ Clearly, either $\left|v_{t} \cdot e_{i}\right|^{2}=1$ or $\left|v_{t} \cdot e_{i}\right|^{2}=4$. If $\left|v_{t} \cdot e_{i}\right|^{2}=1$ then $a_{s} \leq 5$. If $a_{s}<5$ the conclusion follows as in the previous case. If $a_{s}=5$ and $\left|v_{s} \cdot e_{k}\right|>1$ for some $k$, then $v_{s}=a e_{i}+b e_{j}$ with $a^{2}+b^{2}=5$ for some $i, j$. This immediately implies $v_{s} \cdot v_{t}=1,\left|v_{s} \cdot e_{i}\right|=1$ and $V_{s} \cap V_{t}=\{i\}$, and one gets a contradiction eg by replacing $v_{s}$ with $\pi_{e_{i}}\left(v_{s}\right)$ and $v_{t}$ with $\pi_{e_{i}}\left(v_{t}\right)$. If $\left|v_{t} \cdot e_{i}\right|^{2}=4$ then $a_{s}=2$. But then Proposition 4.5 is incompatible with the assumption $b\left(S_{2}\right)=1$.

The following proposition shows that good subsets with no bad components, possibly disconnected intersection graphs and sufficiently negative invariant $I(S)$ can be contracted to subsets having the same properties. This fact will quickly lead to the main result of this section, ie Corollary 5.4.

Proposition 5.3 Suppose that $n \geq 4$, and let

$$
S=\left\{v_{1}, \ldots, v_{n}\right\} \subseteq \mathbf{D}^{n}=\left\langle e_{1}, \ldots, e_{n}\right\rangle
$$

be a good subset with no bad components such that $I(S)<0$. Then, for some $i, s, t$ the set

$$
S^{\prime}=S \backslash\left\{v_{s}, v_{t}\right\} \cup\left\{\pi_{e_{i}}\left(v_{t}\right)\right\} \subseteq\left\langle e_{1}, \ldots, e_{i-1}, e_{i+1}, \ldots, e_{n}\right\rangle \cong \mathbf{D}^{n-1}
$$

is good and has no bad components. Moreover, either

$$
\left(I\left(S^{\prime}\right), c\left(S^{\prime}\right)\right)=(I(S), c(S))
$$

or

$$
I\left(S^{\prime}\right) \leq I(S)-1 \text { and } c\left(S^{\prime}\right) \leq c(S)+1
$$

Proof Since $I(S)<0$, by Proposition 5.2 we have $\left|v_{i} \cdot e_{j}\right| \leq 1$ for every $i$ and $j$. Moreover, inequality (2-2) holds by Lemma 2.5, hence either $p_{1}(S)>0$ or $p_{2}(S)>0$. If $p_{1}(S)>0$ then the conclusion holds by Proposition 3.3. Therefore from now 
on we shall assume $p_{1}(S)=0$ and $p_{2}(S)>0$. Under this assumption there exist $i, s, t \in\{1, \ldots, n\}$ such that $E_{i}^{S}=\{s, t\}$. If $a_{s}>2$ and $a_{t}>2$, since $S$ has no bad component the hypotheses of Lemma 4.3 are satisfied and either Lemma 4.3(1) or Lemma 4.3(2) holds. If Lemma 4.3(2) holds then so does Lemma 4.2(3). But this is impossible because the proof of Lemma 4.2 (see the fourth case) shows that if $V_{s}=\{i, j\}$ then $V_{t} \supseteq\{i, j\}$, which is incompatible with $v_{s} \cdot v_{t}=1$ and $\left|v_{t} \cdot e_{j}\right| \leq 1$ for every $j$. If the conclusion of Lemma 4.3(1) holds then the set

$$
S^{\prime}=S \backslash\left\{v_{s}, v_{t}\right\} \cup\left\{\pi_{e_{i}}\left(v_{t}\right)\right\}
$$

is good and clearly $I\left(S^{\prime}\right) \leq I(S)-1$ and $c\left(S^{\prime}\right) \leq c(S)+1$. Now we argue that $S^{\prime}$ has no bad components.

First, we claim that if $S^{\prime}$ has a bad component $C^{\prime} \subseteq S^{\prime}$ then, if $\pi: S \backslash\left\{v_{S}\right\} \rightarrow S^{\prime}$ denotes the natural map, $v_{t} \in C:=\pi^{-1}\left(C^{\prime}\right)$. Observe that if $v_{t} \notin C$ then $v_{s}$ must be orthogonal to $C$ (ie to every element of $C$ ). Otherwise, it is easy to check that $v_{s}$ would have nontrivial intersection with at least 2 vectors $e_{j}$ orthogonal to $S \backslash C$. But then, adding to $S_{1}$ the vector obtained from $v_{s}$ by eliminating all the vectors $e_{j}$ which are not orthogonal to $S \backslash C$ would give a contradiction via rank considerations. We conclude that if $v_{t} \notin C$ then $v_{s}$ must be orthogonal to $C$. But then if $S^{\prime}$ has a bad component also $S$ has one, so we get a contradiction. Therefore $v_{t} \in C$.

Next, we observe that by the proof of Proposition 5.2 we have $-4 \leq v_{s} \cdot v_{s} \leq-3$. Using this fact together with $\left|v_{s} \cdot e_{j}\right|=1$ for every $j$ it is now a simple exercise to find a contradiction by analyzing separately the following three cases. We sketch the argument for each case.

First case $\left(v_{s}\right.$ orthogonal to $\left.C\right)$ Since $v_{s} \cdot v_{t}=0, V_{s} \cap V_{t} \supseteq\{i, j\}$ for some index $j$. Moreover, $\left|V_{s} \cap V_{t}\right|$ must be even, therefore it is either 2 or 4 . But if $\left|V_{s} \cap V_{t}\right|=4$ one immediately gets a contradiction from the fact that $v_{s}$ is orthogonal to $C$. Therefore, $\left|V_{s} \cap V_{t}\right|=2$ and $e_{j} \cdot v^{\prime} \neq 0$ for some $v^{\prime} \in C \backslash\left\{v_{t}\right\}$. Since $E_{i}^{S}=\{s, t\}$ and $v_{s} \cdot v^{\prime}=0$, for some $k \neq i, j$ we have $k \in V_{s}$ and $e_{k} \cdot v^{\prime} \neq 0$. It is now easy to see that $e_{k} \cdot v^{\prime \prime} \neq 0$ for some $v^{\prime \prime} \in C \backslash\left\{v_{t}, v^{\prime}\right\}$. Since $v_{s} \cdot v^{\prime \prime}=0$, there is an index $h \neq i, j, k$ such that $k \in V_{s}$ and $e_{k} \cdot v^{\prime \prime} \neq 0$. Since $\left|V_{s}\right| \leq 4$, continuing in this way clearly leads to a contradiction.

Second case ( $v_{s}$ not orthogonal to $C$ but $v_{s} \cdot v_{t}=0$ ) As in the previous case, $V_{s} \cap V_{t}$ has either 2 or 4 elements. But $\left|V_{s} \cap V_{t}\right|=4$ easily leads to a contradiction, therefore $V_{s} \cap V_{t}=\{i, j\}$. Let $v_{r} \in C$ with $v_{s} \cdot v_{r}=1$. We have $V_{s} \cap V_{r} \neq\{j\}$ (otherwise a contradiction follows immediately). If $V_{s}=\{i, j, h\}$ then a contradiction follows quickly by considering the vectors of $C$ which intersect non-trivially $e_{h}$. If 
$V_{s}=\{i, j, h, k\}$ one gets a contradiction via a rank counting argument by replacing $v_{s}$ with $\pi_{e_{j}}\left(\pi_{e_{i}}\left(v_{s}\right)\right)$ and $v_{t}$ with $\pi_{e_{i}}\left(v_{t}\right)$.

Third case $\left(v_{s} \cdot v_{t}=1\right)$ If $V_{s} \cap V_{t}=\{i\}$ then replacing $v_{s}$ with $\pi_{e_{i}}\left(v_{s}\right)$ and $v_{t}$ with $\pi_{e_{i}}\left(v_{t}\right)$ one gets a contradiction by the usual rank counting argument. Therefore $V_{s} \cap V_{t}=\{i, j, k\}$. Again, this gives a contradiction by looking at the vectors of $C$ which intersect non-trivially $e_{j}$ and $e_{k}$.

The previous arguments show that if $a_{s}>2$ and $a_{t}>2$ then the statement of the proposition holds. Therefore we may now assume that for each $i, s, t \in\{1, \ldots, n\}$ such that $E_{i}^{S}=\{s, t\}$ we have either $a_{s}=2$ or $a_{t}=2$. By Lemma 4.4, for at least one choice of $i, s, t$ we have $a_{s}=2$ and either $v_{s}$ is not internal or $v_{s} \cdot v_{t}=1$. Therefore, since $S$ has no bad component the conclusion of either Lemma 4.2(1) or Lemma 4.2(3) holds. But, as we pointed out above, the conclusion of Lemma 4.2(3) leads to a contradiction, therefore Lemma 4.2(1) must hold. Thus, the resulting $S^{\prime}$ has no bad components and, since $\left|v_{t} \cdot e_{i}\right|=1$ and $v_{s}$ is not internal, we have $I\left(S^{\prime}\right)=I(S)$ and $c\left(S^{\prime}\right)=c(S)$.

Corollary 5.4 Suppose that $n \geq 3$, and let $S_{n}=\left\{v_{1}, \ldots, v_{n}\right\} \subseteq \mathbf{D}^{n}$ be a good subset with no bad components and such that $I\left(S_{n}\right)<0$. Then $I\left(S_{n}\right) \in\{-1,-2,-3\}$, there exists a sequence of contractions

$$
S_{n} \searrow S_{n-1} \searrow \cdots \searrow S_{3}
$$

such that, for each $k=3, \ldots, n-1$ the set $S_{k}$ is good, has no bad components and we have either

$$
\left(I\left(S_{k}\right), c\left(S_{k}\right)\right)=\left(I\left(S_{k+1}\right), c\left(S_{k+1}\right)\right)
$$

or

$$
I\left(S_{k}\right) \leq I\left(S_{k+1}\right)-1 \quad \text { and } \quad c\left(S_{k}\right) \leq c\left(S_{k+1}\right)+1 .
$$

Moreover:

(1) If $p_{1}\left(S_{n}\right)>0$ then $I\left(S_{n}\right)=-3, S_{n}$ is standard and one can choose the above sequence so that $I\left(S_{k}\right)=-3$ and $S_{k}$ is standard for every $k=3, \ldots, n-1$ and

(2) If $I\left(S_{n}\right)+c\left(S_{n}\right) \leq 0$ then $S_{3}$ is given, up to applying an automorphism of $\mathbf{D}^{3}$, by either (1) or (2) in Lemma 2.4; if $I\left(S_{n}\right)+c\left(S_{n}\right)<0$ then the former case occurs.

Proof If $n=3$ the corollary follows immediately from Lemma 2.4, so we may assume $n \geq 4$. Since $I\left(S_{n}\right) \leq-c\left(S_{n}\right)<0$, by Lemma 2.5 either $p_{1}\left(S_{n}\right)>0$ or $p_{2}\left(S_{n}\right)>0$. If $p_{1}\left(S_{n}\right)>0$ then the existence of the required sequence as well as (1) follow from 
Corollary 3.5. Moreover, in this case (2) follows from (1) because, by Lemma 2.4, $S_{3}$ is given, up to the action of $\operatorname{Aut}\left(\mathbf{D}^{3}\right)$, by Lemma 2.4(1).

If $p_{1}\left(S_{n}\right)=0$ and $p_{2}\left(S_{n}\right)>0$ the existence of the sequence (5-2) follows from several applications of Proposition 5.3. Since $I\left(S_{k}\right) \leq I\left(S_{k+1}\right)$ for $k=3, \ldots, n-1$, $I\left(S_{n}\right) \in\{-1,-2,-3\}$. If $I\left(S_{n}\right)+c\left(S_{n}\right) \leq 0$, since

$$
I\left(S_{3}\right)+c\left(S_{3}\right) \leq I\left(S_{4}\right)+c\left(S_{4}\right) \leq \cdots \leq I\left(S_{n}\right)+c\left(S_{n}\right) \leq 0,
$$

it follows from Lemma 2.4 that, up to applying an automorphism of $\mathbf{D}^{3}, S_{3}$ must be either of type Lemma 2.4(1) or Lemma 2.4(2). Inequalities (5-3) imply that if $I\left(S_{n}\right)+c\left(S_{n}\right)<0$ then $I\left(S_{3}\right)+c\left(S_{3}\right) \leq-1$, hence $S_{3}$ is given, up the action of Aut $\left(\mathbf{D}^{3}\right)$, by Lemma 2.4(1).

\section{Standard subsets}

Outline In this section we finally look at the subsets of $\mathbf{D}^{n}$ we are mostly interested in, that is the standard subsets with $I(S)<0$. By Corollary 5.4 such subsets satisfy $I(S) \in\{-1,-2,-3\}$. As it turns out, the case $I(S)=-3$ is the easiest, so we deal with this case first in Proposition 6.1. Theorem 6.4 is the main result and the culmination of all the work done in this section and in the previous three sections. It is the main algebraic result underlying the proof of Theorem 1.2. Proposition 6.1 and Theorem 6.4 will be used in the next section to characterize the strings $\left(a_{1}, \ldots, a_{n}\right)$ associated to standard subsets $S \subset \mathbf{D}^{n}$ with $I(S)<0$.

Proposition 6.1 Let $n \geq 3$, and let

$$
S_{n}=\left\{v_{1}, \ldots, v_{n}\right\} \subseteq \mathbf{D}^{n}=\left\langle e_{1}, \ldots, e_{n}\right\rangle
$$

be a standard subset such that $I\left(S_{n}\right)=-3$. Then, there is a sequence of contractions

$$
S_{n} \searrow \cdots \searrow S_{3}
$$

with $I\left(S_{k}\right)=-3$ and $S_{k}$ standard for $k=3, \ldots, n$. Moreover,

(1) $p_{1}\left(S_{n}\right)=p_{2}\left(S_{n}\right)=1$ and $p_{3}\left(S_{n}\right)=n-2$;

(2) If $E_{i}^{S_{n}}=\{s\}$ then $v_{s}$ is internal and $v_{s} \cdot v_{s}=-2$;

(3) If $\left|E_{j}^{S_{n}}\right|=2$ then $E_{j}^{S_{n}}=\{1, n\}$;

(4) either $v_{1} \cdot v_{1}=-2$ or $v_{n} \cdot v_{n}=-2$. 
Proof We argue by induction on $n$. For $n=3$ the statement of the proposition follows immediately from Lemma 2.4 , because $I\left(S_{3}\right)=-3$ implies that $S_{3}$ is given, up to the action of $\operatorname{Aut}\left(\mathbf{D}^{3}\right)$, by Lemma 2.4(1). Let us now assume $n>3$. By Corollary 5.4 there is a sequence of contractions

$$
S_{n} \searrow \cdots \searrow S_{3}
$$

with $I\left(S_{n}\right) \geq \cdots \geq I\left(S_{3}\right)$. Since by Lemma $2.4 I\left(S_{3}\right) \geq-3$, the assumption $I\left(S_{n}\right)=$ -3 implies $I\left(S_{n}\right)=\cdots=I\left(S_{3}\right)=-3$. By Corollary 5.4 it follows that each $S_{k}$ is standard for $k=3, \ldots, n$. Up to applying an element of $\operatorname{Aut}\left(\mathbf{D}^{n}\right)$ we have

$$
S_{n-1}=S_{n} \backslash\left\{v_{s}, v_{t}\right\} \cup\left\{\pi_{e_{n}}\left(v_{t}\right)\right\}
$$

for some $s, t$ with $v_{s}$ final and $v_{s} \cdot v_{s}=-2$. Moreover, we may assume without loss that $v_{s}=e_{1}+e_{n}$. Then, it is easy to check that $\left|E_{1}^{S_{n-1}}\right|=2$ and therefore, by the induction hypothesis, $E_{1}^{S_{n-1}}=\{1, n-1\}$. It follows immediately that $\left|E_{1}^{S_{n}}\right|=3$ and $E_{n}^{S_{n}}=\{1, n\}$, and using this it is very easy to verify the statement of the proposition for $S_{n}$.

The next two lemmas will be used in the proof of Theorem 6.4.

Lemma 6.2 Let $S_{3} \nearrow \ldots \nearrow S_{n}$ be a sequence of expansions such that, for each $k=3, \ldots, n, S_{k}$ is good, has no bad component and $\left(I\left(S_{k}\right), c\left(S_{k}\right)\right)=(-2,2)$. Then,

(1) $p_{1}\left(S_{n}\right)=0, p_{2}\left(S_{n}\right)=2$ and $p_{3}\left(S_{n}\right)=n-2$,

(2) If $E_{i}^{S_{n}}=\left\{t, t^{\prime}\right\}$ then $v_{t}$ and $v_{t^{\prime}}$ are not internal and exactly one of them has square -2 and

(3) If $v_{t} \in S_{n}$ is not internal then there exists $i \in V_{t}$ such that $\left|E_{i}^{S_{n}}\right|=2$.

Proof We argue by induction on $n \geq 3$. For $n=3$ the statement of the lemma follows immediately from Lemma 2.4 , because $I\left(S_{3}\right)=-2$ implies that $S_{3}$ is given, up to the action of $\operatorname{Aut}\left(\mathbf{D}^{3}\right)$, by Lemma 2.4(2). Now we assume $n>3$. Up to applying an element of $\operatorname{Aut}\left(\mathbf{D}^{n}\right)$ we have

$$
S_{n-1}=S_{n} \backslash\left\{v_{s}, v_{t}\right\} \cup\left\{\pi_{e_{n}}\left(v_{t}\right)\right\}
$$

for some $s, t$ with $v_{s}$ final and $v_{s} \cdot v_{s}=-2$. As in the proof of Proposition 6.1 we may assume without loss that $v_{s}=e_{1}+e_{n}$, and it is easy to check that $\left|E_{1}^{S_{n-1}}\right|=2$. Using the fact that, by the induction hypothesis, the lemma holds for $S_{n-1}$ it is now easy to check that $\left|E_{1}^{S_{n}}\right|=3$ and $v_{t}$ is not internal, and from this that the lemma holds for $S_{n}$. 
Lemma 6.3 Let $S_{3} \subset \mathbf{D}^{3}$ be a good subset with $I\left(S_{3}\right)=-3$ and $c\left(S_{3}\right)=1$. Suppose that $S_{3} \nearrow \ldots \nearrow S_{k}$ is a sequence of expansions such that, for each $h=3, \ldots, k, S_{h}$ is good, has no bad component and $\left(I\left(S_{h}\right), c\left(S_{h}\right)\right)=(-3,1)$. Then, it is not possible to expand $S_{k}$ by an isolated $(-3)-v e c t o r$.

Proof We may assume that

$$
S_{k}=\left\{v_{1}, \ldots, v_{k}\right\} \subset\left\langle e_{1}, \ldots, e_{k}\right\rangle \cong \mathbf{D}^{k} .
$$

By contradiction, suppose that $S_{k+1} \subset \mathbf{D}^{k+1}$ is obtained by expanding $S_{k+1}$ via an isolated (-3)-vector $v_{k+1}$. Up to applying an automorphism of $\mathbf{D}^{k+1}$ we can write $v_{k+1}=e_{1}+e_{2}+e_{k+1}$. Since $v_{k+1}$ is isolated and $\left|E_{k+1}^{S_{k+1}}\right|=2$, we have

$$
\begin{aligned}
& \left|E_{1}^{S_{k+1} \backslash\left\{v_{k+1}\right\}}\right|+\left|E_{2}^{S_{k+1} \backslash\left\{v_{k+1}\right\}}\right|+\left|E_{k+1}^{S_{k+1} \backslash\left\{v_{k+1}\right\}}\right| \\
& \quad=\left|E_{1}^{S_{k}}\right|+\left|E_{2}^{S_{k}}\right|+1 \equiv \sum_{i \neq k+1} v_{i} \cdot v_{k+1} \quad(\bmod 2) \equiv 0 \quad(\bmod 2) .
\end{aligned}
$$

This shows that the sum $\left|E_{1}^{S_{k}}\right|+\left|E_{2}^{S_{k}}\right|$ must be odd. Therefore by Proposition 6.1 we may assume $E_{1}^{S_{k}}=\{1, k\}$ and either $\left|E_{2}^{S_{k}}\right|=1$ or $\left|E_{2}^{S_{k}}\right|=3$. Since $v_{k+1}$ is orthogonal to each $v_{i}$ for $i=1, \ldots, k$, using Proposition 6.1 it is easy to check that both cases $\left|E_{2}^{S_{k}}\right|=1$ and $\left|E_{2}^{S_{k}}\right|=3$ lead to a contradiction.

Theorem 6.4 Let $n \geq 3$, and let

$$
S_{n}=\left\{v_{1}, \ldots, v_{n}\right\} \subseteq \mathbf{D}^{n}
$$

be a standard subset such that $I\left(S_{n}\right)<0$. Then, $I\left(S_{n}\right) \in\{-1,-2,-3\}$ and there is a sequence of contractions

$$
S_{n} \searrow \cdots \searrow S_{3}
$$

such that for every $k=3, \ldots, n-1$ the set $S_{k}$ is standard and $I\left(S_{k}\right) \leq I\left(S_{k+1}\right)$.

Proof We argue by induction on $n \geq 3$. For $n=3$ the theorem follows immediately from Lemma 2.4, so we assume $n>3$ and that the theorem holds true for sets of cardinality between 3 and $n-1$. By Corollary 5.4 we have $I\left(S_{n}\right) \in\{-1,-2,-3\}$ and there is a sequence of contractions

$$
S_{n} \searrow \cdots \searrow S_{3}
$$

such that for every $k=3, \ldots, n-1$, each $S_{k}$ is good, it has no bad components, and we have either

$$
\left(I\left(S_{k}\right), c\left(S_{k}\right)\right)=\left(I\left(S_{k+1}\right), c\left(S_{k+1}\right)\right)
$$


or

$$
I\left(S_{k}\right) \leq I\left(S_{k+1}\right)-1 \text { and } c\left(S_{k}\right) \leq c\left(S_{k+1}\right)+1 .
$$

If $I\left(S_{n}\right)=-3$ the theorem follows from Proposition 6.1, therefore we may assume $I\left(S_{n}\right) \in\{-2,-1\}$.

Suppose first $I\left(S_{n}\right)=-2$. By Corollary 5.4(2) we have $\left(I\left(S_{3}\right), c\left(S_{3}\right)\right)=(-3,1)$. Then, (6-1) and (6-2) force $c\left(S_{k}\right)=1$ for every $k=3, \ldots, n-1$, therefore the theorem follows in this case.

Now assume $I\left(S_{n}\right)=-1$. By $(6-1)$ and (6-2) we have $c\left(S_{n-1}\right) \leq 2$. If $c\left(S_{n-1}\right)=1$ we can apply the induction hypothesis and immediately obtain the theorem, therefore we may assume $\left(I\left(S_{n-1}\right), c\left(S_{n-1}\right)\right)=(-2,2)$. By Corollary 5.4(2) $\left(I\left(S_{3}\right), c\left(S_{3}\right)\right)$ is equal to either $(-2,2)$ or $(-3,1)$. If $\left(I\left(S_{3}\right), c\left(S_{3}\right)\right)=(-3,1)$, it is easy to check using (6-1) and (6-2) that for some $3 \leq k<n-1$ we have $S_{k+1}=\left\{v_{1}, \ldots, v_{k+1}\right\}$ and

$$
S_{k}=S_{k+1} \backslash\left\{v_{k+1}, v_{t}\right\} \cup\left\{\pi_{e_{k+1}}\left(v_{t}\right)\right\},
$$

where $I\left(S_{k+1}\right)=-2$ and $I\left(S_{k}\right)=-3$. But again by (6-1) and (6-2) we must have

$$
I\left(S_{k}\right)=I\left(S_{k-1}\right)=\cdots=I\left(S_{3}\right)=-3
$$

and therefore $c\left(S_{k}\right)=c\left(S_{k-1}\right)=\cdots=c\left(S_{3}\right)=1$. This implies that $v_{k+1}$ is isolated and $v_{k+1} \cdot v_{k+1}=-3$, but it contradicts Lemma 6.3. Therefore from now on we assume $\left(I\left(S_{3}\right), c\left(S_{3}\right)\right)=(-2,2)$.

The contraction $S_{n} \searrow S_{n-1}$ involves eliminating an internal vector of square -3 , while the sequence of contractions

$$
S_{n-1} \searrow \cdots \searrow S_{3}
$$

satisfies the assumptions of Lemma 6.2. Let us write

$$
S_{n-1}=S_{n} \backslash\left\{v_{s}, v_{t}\right\} \cup\left\{\pi_{e_{i}}\left(v_{t}\right)\right\} \subseteq\left\langle e_{1}, \ldots, e_{i-1}, e_{i+1}, \ldots, e_{n}\right\rangle \cong \mathbf{D}^{n-1}
$$

for some $i, 1<s<n$ and $t \neq s$ with $a_{s}=3$. Up to applying an automorphism of $\mathbf{D}^{n}$ we may assume $i=n$ and $v_{s}=e_{1}+e_{2}+e_{n}$. Moreover, we can write $S_{n-1}$ as a union $S_{n-1}=S_{n-1}^{1} \cup S_{n-1}^{2}$ of subsets with connected intersection graphs, where

$$
S_{n-1}^{1}=\left\{v_{1}^{\prime}, \ldots, v_{s-1}^{\prime}\right\}, \quad S_{n-1}^{2}=\left\{v_{s+1}^{\prime}, \ldots, v_{n}^{\prime}\right\} .
$$


In view of Proposition 5.2 it is easy to check that, since $v_{S-1} \cdot v_{s}=v_{S+1} \cdot v_{s}=1$ and $\left|E_{n}^{S_{n}}\right|=2$, we have

$$
\begin{aligned}
2 & =v_{s-1} \cdot v_{s}+v_{s+1} \cdot v_{s}=\sum_{i \neq s} v_{i} \cdot v_{s} \\
& \equiv\left|E_{1}^{S_{n} \backslash\left\{v_{s}\right\}}\right|+\left|E_{2}^{S_{n} \backslash\left\{v_{s}\right\}}\right|+\left|E_{n}^{S_{n} \backslash\left\{v_{s}\right\}}\right| \quad(\bmod 2) \\
& \equiv\left|E_{1}^{S_{n-1}}\right|+\left|E_{2}^{S_{n-1}}\right|+1 \quad(\bmod 2),
\end{aligned}
$$

and therefore the sum $\left|E_{1}^{S_{n-1}}\right|+\left|E_{2}^{S_{n-1}}\right|$ must be odd. On the other hand, by Lemma $6.2\left|E_{i}^{S_{n-1}}\right|$ is equal to either 2 or 3 for every $i$. Therefore, we may assume $\left|E_{1}^{S_{n-1}}\right|=$ 2 and $\left|E_{2}^{S_{n-1}}\right|=3$. Moreover, by Lemma 6.2 we may also assume $\left|E_{3}^{S_{n-1}}\right|=2$ and $E_{1}^{S_{n-1}} \cup E_{3}^{S_{n-1}}=\{1, s-1, s+1, n\}$. Therefore, if $e_{1} \in V_{s-1} \cap V_{s+1}$ then $e_{3} \in V_{1} \cap V_{n}$. In this case, by Lemma 6.2 either $v_{1} \cdot v_{1}=-2$ and $v_{n} \cdot v_{n}<-2$ or $v_{n} \cdot v_{n}=-2$ and $v_{1} \cdot v_{1}<-2$. By symmetry we may assume the latter occurs and define

$$
S_{n-1}^{\prime}:=S_{n} \backslash\left\{v_{1}, v_{n}\right\} \cup\left\{\pi_{e_{3}}\left(v_{1}\right)\right\} .
$$

Clearly $\left(I\left(S_{n-1}^{\prime}\right), c\left(S_{n-1}^{\prime}\right)\right)=(-1,1)$ and $S_{n-1}^{\prime}$ is obtained from $S_{n}$ by a contraction, hence applying the induction hypothesis to $S_{n-1}^{\prime}$ we get the statement of the theorem. Thus, by symmetry and Lemma 6.2 we may assume $e_{1} \in V_{s-1} \cap V_{n}$. If $e_{n} \in V_{s+1}$ then, since $v_{s} \cdot v_{s+1}=1, e_{2} \notin V_{s+1} \cup V_{s-1}$. But this conflicts with $\left|E_{1}^{S_{n-1}}\right|=2$ and $\left|E_{2}^{S_{n-1}}\right|=3$, therefore $e_{n} \notin V_{s+1}, e_{2} \in V_{s+1}$ and $e_{3} \in V_{1} \cap V_{s+1}$. If $v_{1} \cdot v_{1}=-2$ then by Lemma $6.2 v_{s+1} \cdot v_{s+1}<-2$, we can define

$$
S_{n-1}^{\prime}:=S_{n} \backslash\left\{v_{1}, v_{s+1}\right\} \cup\left\{\pi_{e_{3}}\left(v_{s+1}\right)\right\}
$$

and argue as before. If $v_{1} \cdot v_{1}<-2$ then $v_{s+1} \cdot v_{s+1}=-2$ and therefore $V_{s+1}=\left\{e_{2}, e_{3}\right\}$. Since $v_{1} \cdot v_{s+1}=0$ and $e_{3} \in V_{1}$, this implies $e_{2} \in V_{1}$. Now either $s=2$ and $v_{1}=v_{s-1}$ or $s>2$ and $v_{1} \cdot v_{s-1}=0$. In the former case $e_{1} \in V_{1}=V_{s-1}$, and since $v_{1} \cdot v_{s}=1$, we must also have $e_{n} \in V_{1}$. In the latter case we still have $e_{n} \in V_{1}$ because $e_{1} \notin V_{1}$. Therefore in either case we can define

$$
S_{n-1}^{\prime}:=S_{n} \backslash\left\{v_{1}, v_{s}\right\} \cup\left\{\pi_{e_{n}}\left(v_{1}\right)\right\} .
$$

and conclude as before.

\section{Strings associated to standard subsets}

Outline In this section we use Proposition 6.1 and Theorem 6.4 to identify the strings $\left(a_{1}, \ldots, a_{n}\right)$ corresponding to standard subsets $S \subseteq \mathbf{D}^{n}$ with $I(S) \in\{-1,-2,-3\}$. These results will be used in Section 9 to prove Theorem 1.2. 
The case $I=-3$

Recall Notation (2-3).

Lemma 7.1 Let $n \geq 3$ and let $S_{n}=\left\{v_{1}, \ldots, v_{n}\right\} \subseteq \mathbf{D}^{n}$ be a standard subset such that $I\left(S_{n}\right)=-3$. Suppose $v_{i} \cdot v_{i}=-a_{i}$ for $i=1, \ldots, n$. Then, the string $\left(a_{1}, \ldots, a_{n}\right)$ is obtained from $(2,2,2)$ via a finite sequence of operations of the following types:

(1) $\left(s_{1}, s_{2}, \ldots, s_{k-1}, s_{k}\right) \mapsto\left(s_{1}+1, s_{2}, \ldots, s_{k-1}, s_{k}, 2\right)$ and

(2) $\left(s_{1}, s_{2}, \ldots, s_{k-1}, s_{k}\right) \mapsto\left(2, s_{1}, s_{2}, \ldots, s_{k-1}, s_{k}+1\right)$.

It follows that either $\left(a_{1}, \ldots, a_{n}\right)$ or $\left(a_{n}, \ldots, a_{1}\right)$ is of the form

$$
\begin{aligned}
& \left(c_{k}+1,2^{\left[c_{k-1}-1\right]}, c_{k-2}+2, \ldots, c_{3}+2,2^{\left[c_{2}-1\right]},\right. \\
& \left.c_{1}+2,2^{\left[c_{1}+1\right]}, c_{2}+2, \ldots, c_{k-1}+2,2^{\left[c_{k}-1\right]}\right) \text { or } \\
& \left(c_{1}+1,2^{\left[c_{1}+1\right]}\right),
\end{aligned}
$$

for some integers $c_{1}, \ldots, c_{k} \geq 1$ and $k \geq 3$.

Proof By Proposition 6.1 there is a sequence of expansions

$$
S_{3} \nearrow \cdots \nearrow S_{n}
$$

with $S_{3}$ given, up to applying an element of $\operatorname{Aut}\left(\mathbf{D}^{3}\right)$, by Lemma 2.4(1) and each expansion is obtained by adding a final vector of square -2 while simultaneously decreasing by 1 the square of the opposite final vector. This immediately implies the first part statement. The second part of the statement follows from a straightforward calculation.

\section{The case $I=-2$}

Lemma 7.2 Let $n \geq 4$, and let $S_{n}=\left\{v_{1}, \ldots, v_{n}\right\} \subseteq \mathbf{D}^{n}$ be a standard subset such that $I\left(S_{n}\right)=-2$. Suppose $v_{i} \cdot v_{i}=-a_{i}$ for $i=1, \ldots, n$. Then, either $\left(a_{1}, \ldots, a_{n}\right)$ or $\left(a_{n}, \ldots, a_{1}\right)$ is of one of the following types:

(1) $\left(2^{[t]}, 3,2+s, 2+t, 3,2^{[s]}\right), s, t \geq 0$ or

(2) $\left(2^{[t]}, 3+s, 2,2+t, 3,2^{[s]}\right), s, t \geq 0$.

Proof By Theorem 6.4 and Lemma 2.4 there is a sequence of contractions

$$
S_{n} \searrow \cdots \searrow S_{k} \searrow S_{k-1} \searrow \cdots \searrow S_{3}
$$


of standard subsets with $I\left(S_{3}\right)=-3$ and therefore, for some $n \geq k>3, I\left(S_{n}\right)=$ $\cdots=I\left(S_{k}\right)=-2$ and $I\left(S_{k-1}\right)=\cdots=I\left(S_{3}\right)=-3$. Moreover, we may assume $S_{k}=\left\{v_{1}, \ldots, v_{k}\right\}$ and

$$
S_{k-1}=S_{k} \backslash\left\{v_{k}, v_{t}\right\} \cup\left\{\pi_{e_{k}}\left(v_{t}\right)\right\} \subseteq\left\langle e_{1}, \ldots, e_{k-1}\right\rangle \cong \mathbf{D}^{k-1}
$$

for some $1 \leq t \leq k-1$ and $v_{k} \cdot v_{k}=-3$, with $v_{k}$ final. Up to applying an element of $\operatorname{Aut}\left(\mathbf{D}^{k}\right)$ we may also assume that $v_{k}=e_{1}+e_{2}+e_{k}$. Moreover, by Proposition 6.1 we have $p_{1}\left(S_{k-1}\right)=1$. Therefore, if $\left|E_{1}^{S_{k-1}}\right|>1$ and $\left|E_{2}^{S_{k-1}}\right|>1$ then we would have $p_{1}\left(S_{k}\right)>0$, which would imply $I\left(S_{k}\right)=-3$ by Corollary 5.4(1). Since $v_{k}$ is final, it is easy to see, as in the proof of Theorem 6.4, that the number $\left|E_{1}^{S_{k-1}}\right|+\left|E_{2}^{S_{k-1}}\right|$ must be even. Thus, in view of Proposition 6.1 we may assume $\left|E_{1}^{S_{k-1}}\right|=1$ and

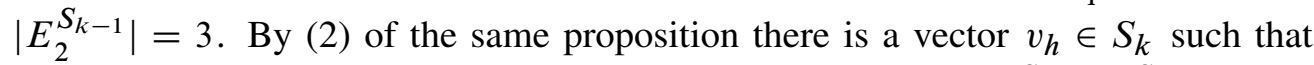
$1<h<k-1$ and $e_{1} \cdot v_{h} \neq 0$. If $t=h$ then $v_{h} \cdot e_{k} \neq 0$, therefore $E_{1}^{S_{k}}=E_{k}^{S_{k}}=\{h, k\}$. Since $\left|E_{2}^{S_{k}}\right|=4$, there exists $s \notin\{h, k-1, k\}$ such that $e_{2} \in V_{s}$. But $v_{s} \cdot v_{k}=0$ implies $s \in E_{1}^{S_{k}} \cup E_{k}^{S_{k}}$, which is impossible. Therefore we have $t \neq h, v_{h} \cdot e_{k}=0$ and $e_{2} \in V_{h}$. Then $v_{h} \in\left\{ \pm\left(e_{1}-e_{2}\right)\right\}$, and since $\left|E_{1}^{S_{k-1}}\right|=1$ this implies $e_{2} \in V_{h-1} \cap V_{h+1}$ as well. If $h+1<k-1$ then, since $E_{1}^{S_{k}}=\{h, k\}, v_{h-1} \cdot v_{k}=v_{h+1} \cdot v_{k}=0$ implies $e_{k} \in E_{h-1}^{S_{k}} \cap E_{h+1}^{S_{k}}$, which is impossible because $\left|E_{k}^{S_{k}}\right|=2$. Therefore we must conclude $h+1=k-1$ and $e_{k} \in V_{h-1}$.

Combining this analysis with the proof of Proposition 6.1 shows that if $S_{k-1}=$ $\left\{v_{1}^{\prime}, \ldots, v_{k-1}^{\prime}\right\}$ then, up to the action of $\operatorname{Aut}\left(\mathbf{D}^{k-1}\right)$ we have

$$
v_{k-1}^{\prime}=-e_{2}-e_{3}-\cdots-e_{k-1} \text { and } v_{k-i}^{\prime}=e_{i}-e_{i-1}, i=2, \ldots, k-1 .
$$

Moreover, $v_{i}=v_{i}^{\prime}$ for $1 \leq i \leq k-1, i \neq k-3$, and $v_{k-3}=v_{k-3}^{\prime}+e_{k}$. Now we see that $S_{k}$ can be contracted to a standard subset $S_{k-1}^{\prime}$ by dropping $v_{1}$ and replacing $v_{k-1}$ with $v_{k-1}^{(1)}:=\pi_{e_{k-1}}\left(v_{k-1}\right)$. Similarly, for $i=2, \ldots, k-4$ we can define $S_{k-i}^{\prime}$ by dropping $v_{i}$ from $S_{k-i+1}^{\prime}$ and replacing $v_{k-i+1}^{(i-1)}$ with $v_{k-i}^{(i)}:=\pi_{e_{k-i}}\left(v_{k-i+1}^{(i-1)}\right)$. Continuing in this way we can construct a new sequence of contractions

$$
S_{k} \searrow S_{k-1}^{\prime} \searrow \cdots \searrow S_{4}^{\prime}
$$

of standard subsets with $I\left(S_{i}^{\prime}\right)=-2$ for $i=4, \ldots, k-1$, and such that, up to an automorphism of $\mathbf{D}^{4}$,

$$
S_{4}^{\prime}=\left\{e_{3}-e_{2}+e_{4}, e_{2}-e_{1},-e_{2}-e_{3}, e_{1}+e_{2}+e_{4}\right\}
$$


Analysing (7-1) it is easy to see that, up to reversing the $k$-tuple $\left(v_{1}, \ldots, v_{k}\right)$, if $\left|E_{i}^{S_{k}}\right|=2$ then

$$
E_{i}^{S_{k}} \in\{\{1, k-1\},\{k-2, k\},\{k-3, k\}\} .
$$

Since the subset $S_{n}$ is obtained from $S_{k}$ by a sequence of expansions by final (-2)vectors, the lemma follows easily.

\section{The case $I=-1$}

Lemma 7.3 Let $n \geq 4$ and let $S_{n}=\left\{v_{1}, \ldots, v_{n}\right\} \subseteq \mathbf{D}^{n}$ be a standard subset such that $I\left(S_{n}\right)=-1$. Suppose $v_{i} \cdot v_{i}=-a_{i}$ for $i=1, \ldots, n$. Then, either $\left(a_{1}, \ldots, a_{n}\right)$ or $\left(a_{n}, \ldots, a_{1}\right)$ is of one of the following types:

(1) $\left(t+2, s+2,3,2^{[t]}, 4,2^{[s]}\right), s, t \geq 0$,

(2) $\left(t+2,2,3+s, 2^{[t]}, 4,2^{[s]}\right), s, t \geq 0$ or

(3) $\left(3+t, 2,3+s, 3,2^{[t]}, 3,2^{[s]}\right), s, t \geq 0$.

Proof By Theorem 6.4 and Lemma 2.4 there is a sequence of contractions

$$
S_{n} \searrow \cdots \searrow S_{3}
$$

of standard subsets with $I\left(S_{3}\right)=-3$. Thus, either for some $3<k \leq n$ we have

$$
I\left(S_{n}\right)=\cdots=I\left(S_{k}\right)=-1, \quad I\left(S_{k-1}\right)=\cdots=I\left(S_{3}\right)=-3,
$$

or for some $3 \leq k<h \leq n$ we have

$$
\begin{aligned}
I\left(S_{n}\right) & =\cdots=I\left(S_{h}\right)=-1, \quad I\left(S_{h-1}\right)=\cdots=I\left(S_{k}\right)=-2, \\
I\left(S_{k-1}\right) & =\cdots=I\left(S_{3}\right)=-3 .
\end{aligned}
$$

First case ((7-2) holds) The expansion $S_{k-1} \nearrow S_{k}$ is obtained by adding a final $(-4)$-vector which can be assumed of the form $v_{k}=e_{1}+e_{2}+e_{3}+e_{k}$, and $p_{1}\left(S_{k}\right)=0$ otherwise by Corollary 5.4(1) $I\left(S_{k}\right)=-3$. Moreover, by Proposition 6.1 we have $p_{1}\left(S_{k-1}\right)=1$ and, by the parity argument used in the proofs of Theorem 6.4 and Lemma 7.2 we have $\left|E_{1}^{S_{k-1}}\right|=1,\left|E_{2}^{S_{k-1}}\right|=2$ and $\left|E_{3}^{S_{k-1}}\right|=3$ (up to renaming the vectors $e_{1}, e_{2}$ and $e_{3}$ ). Also, by Proposition 6.1 we have $e_{1} \in V_{l}$ for some $1<l<k-1$ and $v_{l}$ is of the form $v_{l}= \pm e_{1} \pm e_{i}$ with $E_{i}^{S_{k-1}}=\{l-1, l, l+1\}$. Since $v_{l} \cdot v_{k}=0$ this immediately implies $i=3$. Again by the proposition we have $e_{2} \in V_{1} \cap V_{k-1} \cap V_{k}$. Since $v_{k} \cdot v_{1}=v_{k} \cdot v_{l-1}=0$, it is easy to check that if $l-1 \neq 1$ then $e_{k} \in V_{l-1} \cap V_{1}$, which is impossible because $\left|E_{k}^{S_{k}}\right|=2$. Therefore $l=2, e_{3} \in V_{1}$ and $e_{k} \in V_{3}$. By 
the proposition this implies that $S_{k}$ has associated string (up to a reflection) of the form

$$
\left(t+2,2,3,2^{[t]}, 4\right),
$$

$E_{1}^{S_{k}}=\{2, k\}, E_{k}^{S_{k}}=\{3, k\}$ and $\left|E_{i}^{S_{k}}\right|>2$ for $i \neq 1, k$. Since $S_{n}$ is obtained from $S_{k}$ by a sequence of expansions obtained by adding final vectors of square -2 , this implies that $S_{n}$ has associated fraction (up to a reflection) as in (1) or (2) from the statement of the lemma.

Second case ((7-3) holds) Arguing as in the proof of Lemma 7.2 we may assume that $k=4$, and

$$
S_{4}=\left\{v_{1}=e_{1}-e_{2}+e_{4}, v_{2}=e_{2}+e_{3}, v_{3}=-e_{2}-e_{1}, v_{4}=e_{4}+e_{2}-e_{3}\right\},
$$

with $S_{4} \nearrow \cdots \nearrow S_{h-1}$ consisting of expansions obtained by adding final (-2)-vectors and the expansion $S_{h-1} \nearrow S_{h}$ obtained by adding a final $(-3)$-vector which we can

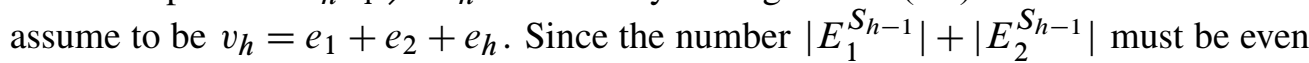
and it can be easily checked that $p_{2}\left(S_{h-1}\right)=3, p_{4}\left(S_{h-1}\right)=1$ and $p_{3}\left(S_{h-1}\right)=h-5$, a case-by-case analysis shows that $\left|E_{1}^{S_{h-1}}\right|=\left|E_{2}^{S_{h-1}}\right|=2$.

This implies, assuming $S_{h}=\left\{v_{1}, \ldots, v_{h}\right\}$, that $i \in E_{1}^{S_{h}} \cap E_{2}^{S_{h}}$ for some $i$ with $i<h$. The same analysis of the sequence $S_{4} \nearrow \ldots \nearrow S_{h-1}$ used at the end of the proof of Lemma 7.2 shows that $\left|E_{i}^{S_{h-1}}\right|=2$ implies $E_{i}^{S_{h-1}} \cap\{1, h-1\} \neq \varnothing$. Since $v_{h-1} \cdot v_{h}=1$, up to renaming $e_{1}$ and $e_{2}$ we may assume $e_{1}, e_{2} \in V_{1}$ and $e_{2} \in V_{h-1}$. It is easy to check that this implies that all the $(-2)$-vectors added in the sequence $S_{4} \nearrow \cdots \nearrow S_{h-1}$ are added from the same side. If they are added eg from the right-hand side the string associated to $S_{h-1}$ has the form

$$
\left(3+t, 2,2,3,2^{[t]}\right) .
$$

Moreover, the same analysis as above shows that $E_{h}^{S_{h}}=\{3, h\}$ and $\left|E_{i}^{S_{h}}\right|=3$ for every $i \in V_{1}$. This implies that the sequence $S_{h} \nearrow \cdots \nearrow S_{n}$ consists of expansions obtained by adding $(-2)$-vectors from the right-hand side only. It follows that the string associated to $S_{n}$ is of the form (3) from the statement of the lemma. If the $(-2)$-vectors added in the sequence $S_{4} \nearrow \ldots \nearrow S_{h-1}$ are added from the left-hand side the resulting string is obtained from (3) by a reflection.

\section{Existence of ribbon surfaces}

Outline In this section we prove the existence of bounding ribbon surfaces for all the 2-bridge links which will occur in the proof of Theorem 1.2 in Section 9. 
We shall use the following elementary fact about continued fractions (see eg [6, Appendix] for a proof). Let $p>q \geq 1$ be coprime integers, and suppose that

$$
\frac{p}{q}=\left[a_{1}, a_{2}, \ldots, a_{n}\right]^{-}, \quad a_{1}, \ldots, a_{h} \geq 2 .
$$

Then,

$$
\frac{p}{q^{\prime}}=\left[a_{n}, a_{n-1}, \ldots, a_{1}\right]^{-},
$$

where $p>q^{\prime} \geq 1$ and $q q^{\prime} \equiv 1(\bmod p)$.

Let $a_{1}, \ldots, a_{2 n}$ be positive integers. The following identity holds (see [9, Proposition 2.3]):

$$
\begin{aligned}
& {\left[a_{1}, \ldots, a_{2 n}\right]^{+}} \\
& = \begin{cases}{\left[a_{1}+1,2^{\left[a_{2}-1\right]}, a_{3}+2,2^{\left[a_{4}-1\right]}, \ldots, a_{2 n-1}+2,2^{\left[a_{2 n}-1\right]}\right]^{-},} & n \geq 2, \\
{\left[a_{1}+1,2^{\left[a_{2}-1\right]}\right]^{-},} & n=1 .\end{cases}
\end{aligned}
$$

Lemma 8.1 Let $p>q \geq 1$ be coprime integers, and suppose that $\frac{p}{q}=\left[a_{1}, \ldots, a_{n}\right]^{-}$, where either $\left(a_{1}, \ldots, a_{n}\right)$ or $\left(a_{n}, \ldots, a_{1}\right)$ is of the form

$$
\begin{aligned}
& \left(c_{k}+1,2^{\left[c_{k-1}-1\right]}, c_{k-2}+2, \ldots, c_{3}+2,2^{\left[c_{2}-1\right]}, c_{1}+2,2^{\left[c_{1}+1\right]}, c_{2}+2, \ldots,\right. \\
& \left.c_{k-1}+2,2^{\left[c_{k}-1\right]}\right) \text { or } \\
& \left(c_{1}+1,2^{\left[c_{1}+1\right]}\right),
\end{aligned}
$$

for some integers $c_{1}, \ldots, c_{k} \geq 1$ and $k \geq 3$. Then, if $p$ is odd $K(p, q)$ bounds an immersed ribbon disk; if $p$ is even the 2 -component link $K(p, q)$ bounds the image under a ribbon immersion of the disjoint union of a disk and a Möbius band.

Proof Let $k, c_{1}, \ldots, c_{k} \geq 1$ be integers. Then, a straightforward application of (8-2) gives

$$
\begin{aligned}
& {\left[c_{k}+1,2^{\left[c_{k-1}-1\right]}, c_{k-2}+2, \ldots, c_{3}+2,2^{\left[c_{2}-1\right]}, c_{1}+2,2^{\left[c_{1}+1\right]}, c_{2}+2, \ldots,\right.} \\
& \left.\left.\quad c_{k-1}+2,2^{\left[c_{k}-1\right]}\right)\right]^{-} \\
& =\left[c_{k}, c_{k-1}, c_{k-2}, \ldots, c_{1}, c_{1}+2, c_{2}, c_{3}, \ldots, c_{k-1}, c_{k}\right]^{+}
\end{aligned}
$$

and

$$
\left[c_{1}+1,2^{\left[c_{1}+1\right]}\right]^{-}=\left[c_{1}, c_{1}+2\right]^{+} .
$$

Recalling that if $0<q^{\prime}<p$ and $q q^{\prime} \equiv 1(\bmod p)$ the link $K\left(p, q^{\prime}\right)$ is isotopic to $K(p, q)$, this shows that the $K(p, q)$ is isotopic to $L\left(c_{k}, \ldots, c_{1}, c_{1}+2, c_{2}, \ldots, c_{k}\right)$ (see Figure 1, case $n$ even). Applying the ribbon move described in the top picture of 
Figure 2 reduces $K(p, q)$ to a 2 -component unlink, as shown in the remaining pictures of Figure 2. By standard facts on ribbon moves, this proves the lemma.

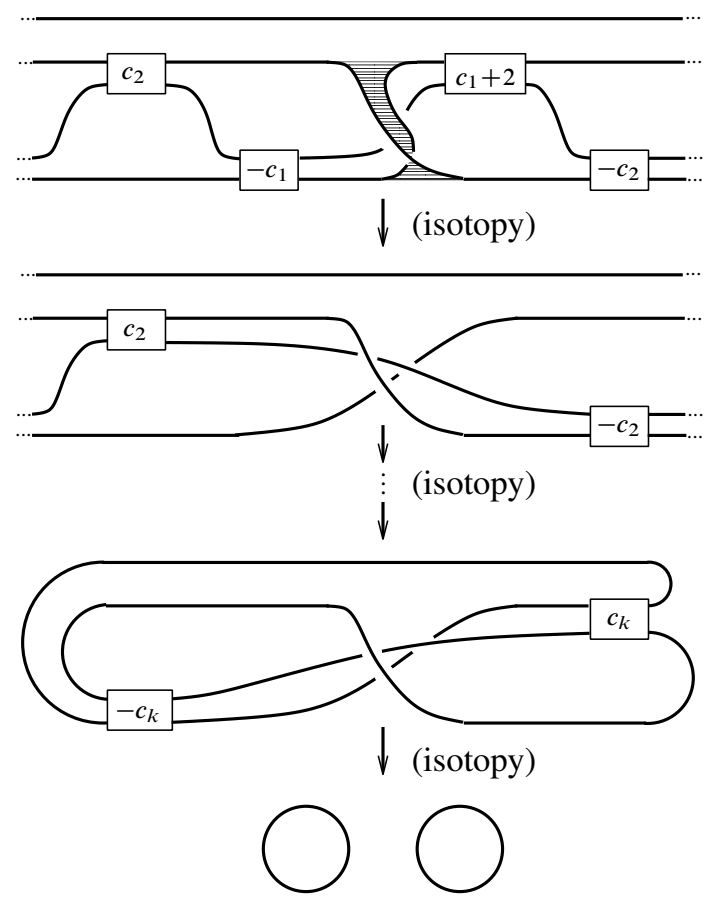

Figure 2: The link $L\left(c_{k}, \ldots, c_{1}, c_{1}+2, \ldots, c_{k}\right)$ bounds a ribbon surface.

Lemma 8.2 Let $L_{a, b}, a, b \in \mathbb{Z}$, be the 2-bridge link given by the top picture of Figure 3. If the link $L_{a, b}$ is a knot then it bounds a ribbon disk. If $L_{a, b}$ has two components then it bounds the image under a ribbon immersion of the disjoint union of a disk and a Möbius band.

Proof Figure 3 shows that after performing two ribbon moves the link $L_{a, b}$ reduces to a 3-component unlink. This proves the lemma.

Lemma 8.3 Let $L_{a, b}^{\prime}, a, b \in \mathbb{Z}$, be the link given by the top picture of Figure 4. If the link $L_{a, b}^{\prime}$ is a knot then it bounds a ribbon disk. If $L_{a, b}^{\prime}$ has two components then it bounds the image under a ribbon immersion of the disjoint union of a disk and a Möbius band. 


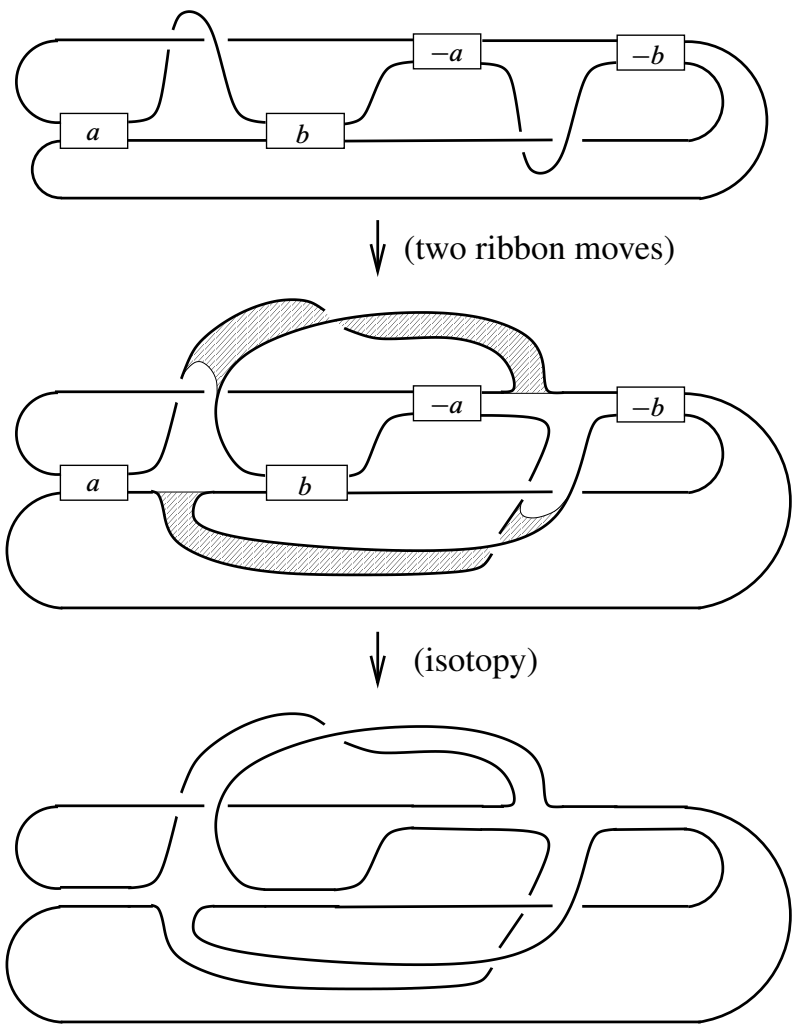

Figure 3: Ribbon moves on the link $L_{a, b}$.

Proof Figure 4 shows that after performing one ribbon move the link $L_{a, b}^{\prime}$ reduces to a 2-component unlink. This proves the lemma.

Lemma 8.4 Let $p>q>0$ be coprime integers, and suppose that $\frac{p}{q}$ is equal to one of the following:

(1) $\left[2^{[t]}, 3, s+2, t+2,3,2^{[s]}\right]^{-}, s, t \geq 0$ or

(2) $\left[2^{[t]}, s+3,2, t+2,3,2^{[s]}\right]^{-}, s, t \geq 0$.

Then, if $p$ is odd $K(p, q)$ bounds a ribbon disk; if $p$ is even the 2-component link $K(p, q)$ bounds the image under a ribbon immersion the disjoint union of a disk and a Möbius band.

Proof By (8-2) we have

$$
[1, t, 1,1, s, 1, t, 1,1, s+1]^{+}=\left[2^{[t]}, 3, s+2, t+2,3,2^{[s]}\right]^{-} .
$$




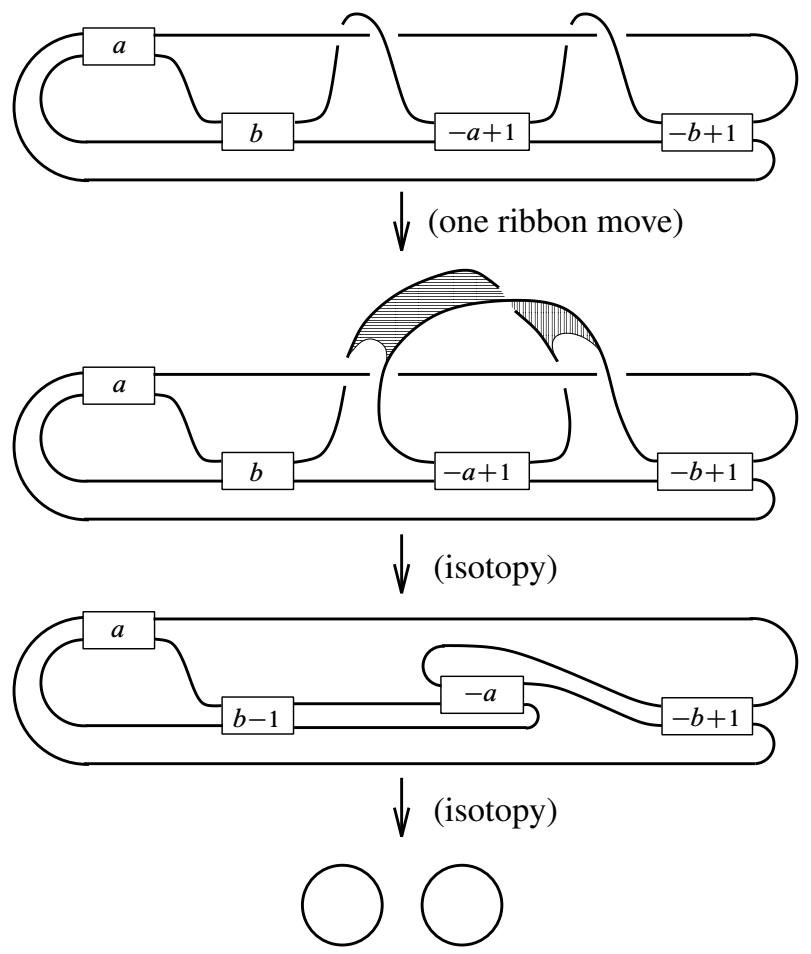

Figure 4: Ribbon move on the link $L_{a, b}^{\prime}$.

Therefore, in Case (1) the link $K(p, q)=L(1, t, 1,1, s, 1, t, 1,1, s+1)$ is given by the top picture in Figure 5. After an isotopy, the knot appears as in the middle picture of Figure 5. After a further isotopy, we obtain the bottom picture of Figure 5. After an isotopy starting with pulling a strand as suggested by the arrow in Figure 5, we arrive at the link given by the top picture of Figure 3 for $(a, b)=(t+2,-s-1)$. Thus, in Case (1) the lemma follows from Lemma 8.2.

By (8-2) we have

$$
[1, t, s+1,2, t, 1,1, s+1]^{+}=\left[2^{[t]}, s+3,2, t+2,3,2^{[s]}\right]^{-} .
$$

Therefore $K(p, q)$ is isotopic to the link $L(1, t, s+1,2, t, 1,1, s+1)$ given in Figure 6. Applying an obvious isotopy it is easy to see that this link is isotopic to the link $L_{t+1,-s-1}^{\prime}$, where $L_{a, b}^{\prime}$, for $a, b \in \mathbb{Z}$, is as in Lemma 8.3. Part (2) of the statement now follows from Lemma 8.3. 

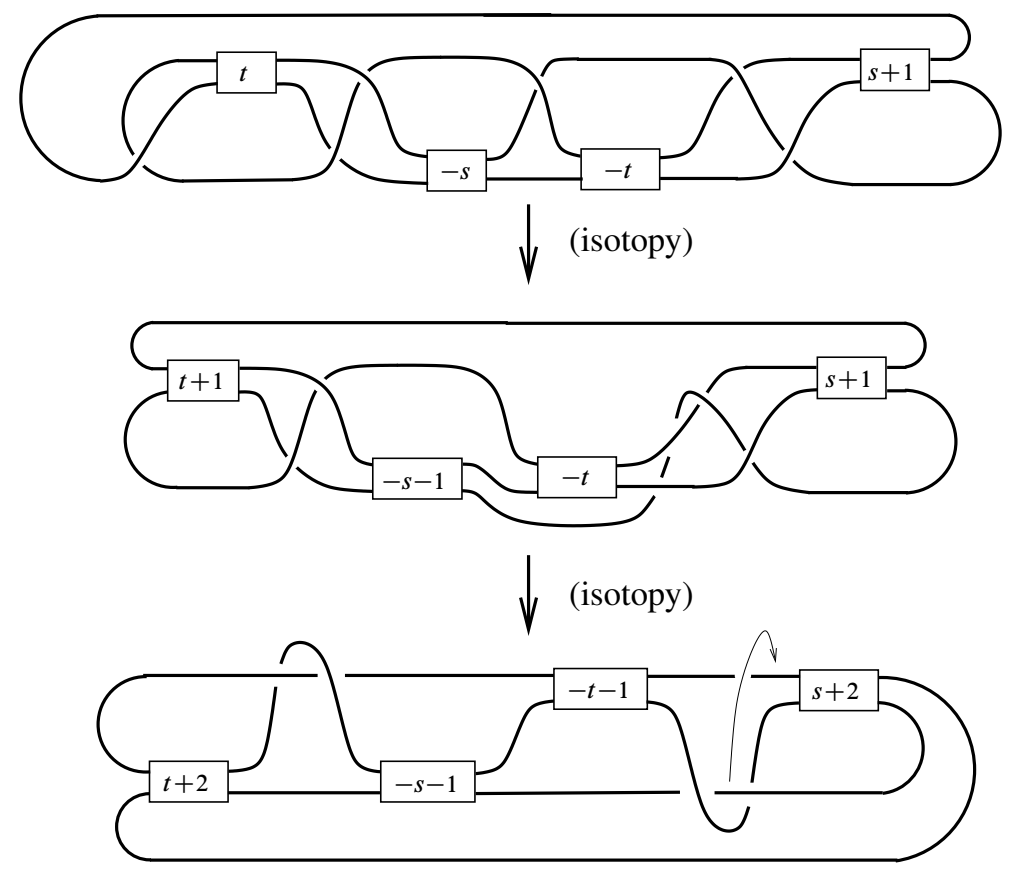

Figure 5: The link $L(1, t, 1,1, s, 1, t, 1,1, s+1)$.

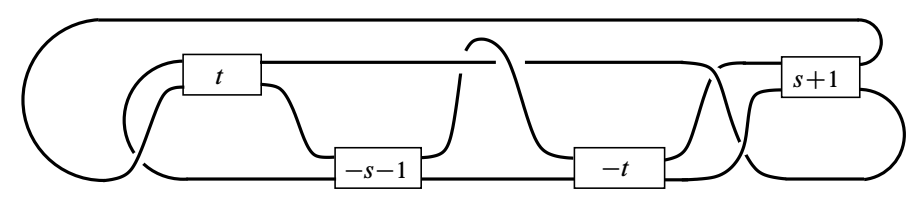

Figure 6: The link $L(1, t, s+1,2, t, 1,1, s+1)$.

Lemma 8.5 Let $p>q \geq 1$ be coprime integers, and suppose that $\frac{p}{q}$ is equal to one of the following:

(1) $\left[t+2, s+2,3,2^{[t]}, 4,2^{[s]}\right]^{-}, s, t \geq 0$,

(2) $\left[t+2,2, s+3,2^{[t]}, 4,2^{[s]}\right]^{-}, s, t \geq 0$ or

(3) $\left[t+3,2, s+3,3,2^{[t]}, 3,2^{[s]}\right]^{-}, s, t \geq 0$.

Then, if $p$ is odd $K(p, q)$ bounds a ribbon disk; if $p$ is even the 2-component link $K(p, q)$ bounds the image under a ribbon immersion of the disjoint union of a disk and a Möbius band. 
Proof By (8-2) we have

$$
[t+1,1, s, 1,1, t+1,2, s+1]^{+}=\left[t+2, s+2,3,2^{[t]}, 4,2^{[s]}\right]^{-}, \quad s, t \geq 0 .
$$

Therefore in Case (1) $K(p, q)$ is isotopic to the link $L(t+1,1, s, 1,1, t+1,2, s+1)$ given by the top picture of Figure 7. Applying the isotopy suggested by the arrow one

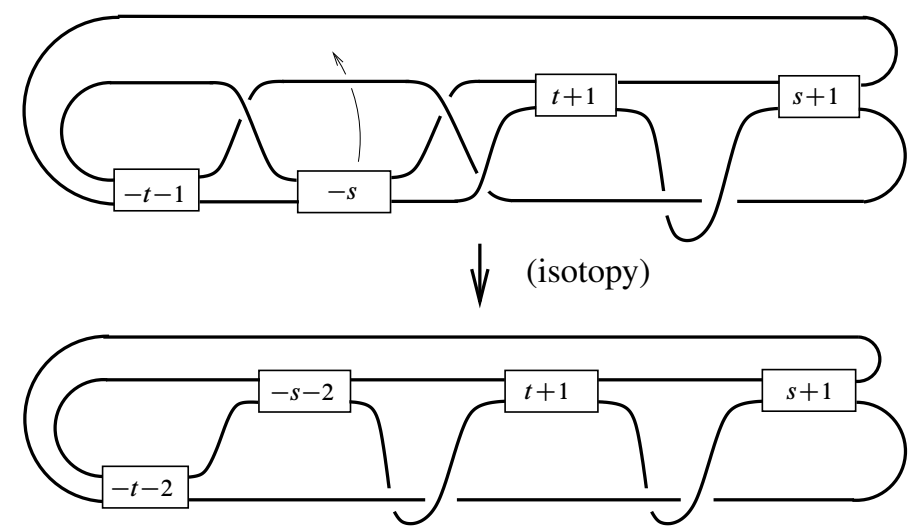

Figure 7: The link $L(t+1,1, s, 1,1, t+1,2, s+1)$.

obtains the link given by the bottom picture of Figure 7, which is easily checked to be the mirror image of the link $L_{t+2, s+2}^{\prime}$, where $L_{a, b}^{\prime}$, for $a, b \in \mathbb{Z}$, is as in Lemma 8.3. Therefore, Part (1) of the statement follows from Lemma 8.3. By (8-2) we have

$$
[t+1,2, s+1, t+1,2, s+1]^{+}=\left[t+2,2,3+s, 2^{[t]}, 4,2^{[s]}\right]^{-}, \quad s, t \geq 0 .
$$

This shows that in Case (2) $K(p, q)$ is isotopic to $L(t+1,2, s+1, t+1,2, s+1)$, which is easily seen to be isotopic to $L_{-t-1,-s-1}$, where $L_{a, b}, a, b \in \mathbb{Z}$ is as in Lemma 8.2. Thus, in Case (2) the statement follows from Lemma 8.2. Now observe that if

$$
\frac{p}{q}=\left[t+3,2, s+3,3,2^{[t]}, 3,2^{[s]}\right]^{-}, \quad s, t \geq 0,
$$

then by Equations (2-4) and (8-1) we have

$$
\frac{p}{p-q^{\prime}}=\left[s+2, t+3,3,2^{[s]}, 4,2^{[t+1]}\right]^{-},
$$

which is of the type considered in Case (1). This concludes the proof.

$\square$

\section{The proof of Theorem 1.2}

Outline In this section we use the results obtained in the previous sections to prove Theorem 1.2. 
Before starting the proof of Theorem 1.2 we need four arithmetic lemmas.

Lemma 9.1 Suppose that $a_{i} \geq 2$ for $i=1, \ldots, n$, are integers and

$$
\left[a_{1}, \ldots, a_{n}\right]^{-}=\frac{m^{2}}{m k \pm 1}, \quad(m, k)=1, \quad 0<k<m .
$$

Then,

$$
\left[2, a_{1}, \ldots, a_{n}, a_{n}+1\right]^{-}=\frac{(2 m-k)^{2}}{(2 m-k) m \pm 1}
$$

and

$$
\left[a_{1}+1, a_{2}, \ldots, a_{n}, 2\right]^{-}=\frac{(m+k)^{2}}{(m+k) k \pm 1} .
$$

Proof Since

$$
(m(m-k) \pm 1)(m k \pm 1) \equiv 1 \quad\left(\bmod m^{2}\right),
$$

by $(8-1)$ we have

$$
\left[a_{n}, \ldots, a_{1}\right]^{-}=\frac{m^{2}}{m(m-k) \pm 1}
$$

therefore

$$
\left[a_{n}+1, \ldots, a_{1}\right]^{-}=\frac{m^{2}+m(m-k) \pm 1}{m(m-k) \pm 1}=\frac{2 m^{2}-m k \pm 1}{m(m-k) \pm 1} .
$$

Similarly, since

$$
(m(m-k) \pm 1)\left(2 m k-k^{2} \pm 2\right)=1-\left(2 m^{2}-m k \pm 1\right)\left(k^{2}-m k \mp 1\right)
$$

we have

$$
\left[a_{1}, \ldots, a_{n}+1\right]^{-}=\frac{2 m^{2}-m k \pm 1}{2 m k-k^{2} \pm 2} .
$$

The first formula in the statement of the lemma now follows by a simple computation. By (9-1) and the first formula in the statement we have

$$
\left[2, a_{n}, \ldots, a_{1}+1\right]^{-}=\frac{(2 m-(m-k))^{2}}{(2 m-m+k) m \pm 1}=\frac{(m+k)^{2}}{(m+k) m \pm 1},
$$

which implies, as before, the second formula in the statement of the lemma.

Lemma 9.2 Let $n \geq 3$ and let $S_{n}=\left\{v_{1}, \ldots, v_{n}\right\} \subseteq \mathbf{D}^{n}$ be a standard subset such that $I\left(S_{n}\right)=-3$. Suppose $v_{i} \cdot v_{i}=-a_{i}$ for $i=1, \ldots, n$. Then,

$$
\left[a_{1}, \ldots, a_{n}\right]^{-}=\frac{m^{2}}{m k+1}
$$

for some integers $m, k$ with $0<k<m$ and $(m, k)=1$.

Proof The fraction associated to the set $S_{3}$ of Lemma 2.4 is $[2,2,2]=4 / 3$, which is of the form $m^{2} /(m+1)$. The lemma follows immediately from Lemma 7.1 and Lemma 9.1. 
In the following proofs we shall use the formula

$$
\left[2^{[t]}, x\right]^{-}=\frac{(t+1) x-t}{t x-(t-1)}, \quad t \in \mathbb{N} \cup\{0\},
$$

which holds for any variable $x$ and can be established by an easy induction.

Lemma 9.3 Let $n \geq 4$, and let $S_{n}=\left\{v_{1}, \ldots, v_{n}\right\} \subseteq \mathbf{D}^{n}$ be a standard subset such that $I\left(S_{n}\right)=-2$. Suppose $v_{i} \cdot v_{i}=-a_{i}$ for $i=1, \ldots, n$. Then, either $\left[a_{1}, \ldots, a_{n}\right]^{-}$or $\left[a_{n}, \ldots, a_{1}\right]^{-}$is of one of the following forms:

(1) $\frac{m^{2}}{m^{2}-d(m-1)}$, where $d$ divides $2 m+1$ or

(2) $\frac{m^{2}}{m^{2}-d(m-1)}$, where $d$ is odd and divides $m-1$.

Proof Using (9-2) one can verify that

$$
\begin{aligned}
& {\left[2^{[t]}, 3, s+2, t+2,3,2^{[s]}\right]^{-}} \\
& \quad=\frac{(2 s t+3 s+3 t+4)^{2}}{(2 s t+3 s+3 t+4)^{2}-(2 s+3)(2 s t+3 s+3 t+3)} .
\end{aligned}
$$

For $m=2 s t+3 s+3 t+4$ and $d=2 s+3$, since $2 m+1=(2 s+3)(2 t+3)$, this shows that the associated fraction is of the form

$$
\frac{m^{2}}{m^{2}-d(m-1)}
$$

where $d$ divides $2 m+1$. Similarly,

$$
\begin{aligned}
{\left[2^{[t]}, s+3,2, t+2,3,2^{[s]}\right]^{-} } & =\frac{(2 s t+2 s+3 t+4)^{2}}{(2 s t+2 s+3 t+4)^{2}-(2 s+3)^{2}(t+1)} \\
& =\frac{m^{2}}{m^{2}-d(m-1)},
\end{aligned}
$$

where $m=2 s t+2 s+3 t+4$ and $d=2 s+3$. Observe that $d$ is odd and divides $m-1=(2 s+3)(t+1)$. By Lemma 7.2 this concludes the proof.

Lemma 9.4 Let $n \geq 4$ and let $S_{n}=\left\{v_{1}, \ldots, v_{n}\right\} \subseteq \mathbf{D}^{n}$ be a standard subset such that $I\left(S_{n}\right)=-1$. Suppose that $v_{i} \cdot v_{i}=-a_{i}, i=1, \ldots, n$. Then, either $\left[a_{1}, \ldots, a_{n}\right]^{-}$or $\left[a_{n}, \ldots, a_{1}\right]^{-}$is of one of the following types:

(1) $\frac{m^{2}}{d(m+1)}$ where $d$ is odd and divides $m+1$;

(2) $\frac{m^{2}}{d(m+1)}$ where $d$ divides $2 m-1$ or

(3) $\frac{m^{2}}{m^{2}-d(m+1)}$ where $d$ is odd and divides $m+1$. 
Proof Using (9-2) one can verify that

$$
\left[t+2, s+2,3,2^{[t]}, 4,2^{[s]}\right]^{-}=\frac{(2 s t+4 s+3 t+5)^{2}}{(2 s+3)^{2}(t+2)}=\frac{m^{2}}{d(m+1)},
$$

where $m=2 s t+4 s+3 t+5$ and $d=2 s+3$ is odd and divides $m+1=(2 s+3)(t+2)$. Similarly,

$$
\left[t+2,2, s+3,2^{[t]}, 4,2^{[s]}\right]^{-}=\frac{(2 s t+3 s+3 t+5)^{2}}{(2 s+3)(2 s t+3 s+3 t+6)}=\frac{m^{2}}{d(m+1)},
$$

where $m=2 s t+3 s+3 t+5$ and $d=2 s+3$ divides $2 m-1=(2 s+3)(2 t+3)$. Finally,

$$
\begin{aligned}
{\left[t+3,2, s+3,3,2^{[t]}, 3,2^{[s]}\right]^{-} } & =\frac{(2 t s+5 s+4 t+9)^{2}}{(s+2)(4 t s+10 s+8 t+17)} \\
& =\frac{m^{2}}{(2 m-1)(m+1) / d},
\end{aligned}
$$

where $m=2 t s+5 s+4 t+9$ and $d=2 t+5$ divides $m+1=(s+2)(2 t+5)$. Since

$$
\frac{(2 m-1)(m+1)}{d}\left(m^{2}-d(m+1)\right) \equiv 1 \quad\left(\bmod m^{2}\right),
$$

by $(8-1)$ we have

$$
\frac{m^{2}}{m^{2}-d(m+1)}=\left[2^{[s]}, 3,2^{[t]}, 3, s+3,2, t+3\right]^{-} .
$$

Thus, the lemma follows by Lemma 7.3.

Proof of Theorem 1.2 We first show that (2) implies (1). Let us assume that (2) holds. Let $\widetilde{\Sigma} \subset B^{4}$ be a smoothly embedded surface obtained by pushing the interior of $\Sigma$ inside the 4-ball. It is easy to check that (regardless of the parity of $p$ ) the inclusion $S^{3} \backslash \partial \widetilde{\Sigma} \subset B^{4} \backslash \widetilde{\Sigma}$ induces a surjective homomorphism

$$
\varphi: H_{1}\left(S^{3} \backslash \partial \widetilde{\Sigma} ; \mathbb{Z}\right) \rightarrow H_{1}\left(B^{4} \backslash \widetilde{\Sigma} ; \mathbb{Z}\right)
$$

such that the homomorphism $H_{1}\left(S^{3} \backslash \partial \widetilde{\Sigma} ; \mathbb{Z}\right) \rightarrow \mathbb{Z} / 2 \mathbb{Z}$ defining the 2-fold cover $L(p, q) \rightarrow S^{3}$ branched along $\partial \widetilde{\Sigma}=K(p, q)$ factors through $H_{1}\left(B^{4} \backslash \widetilde{\Sigma} ; \mathbb{Z}\right)$ via $\varphi$. Therefore, the cover $L(p, q) \rightarrow S^{3}$ extends to a 2-fold cover $W \rightarrow B^{4}$ branched along $\widetilde{\Sigma}$. We may assume that the distance function from the origin $B^{4} \rightarrow[0,1]$ restricted to $\widetilde{\Sigma}$ is a proper Morse function with only index -0 and index -1 critical points. This implies that $W$ has a handlebody decomposition with only $0-, 1-$ and 2-handles (see eg [3, lemma at pages 30-31]). Therefore, from

$$
b_{0}(W)-b_{1}(W)+b_{2}(W)=\chi(W)=2 \chi\left(B^{4}\right)-\chi(\widetilde{\Sigma})=1
$$

we deduce $b_{1}(W)=b_{2}(W)$. On the other hand, since $b_{1}(\partial W)=0$ and $H_{1}(W, \partial W ; \mathbb{Q}) \cong$ $H^{3}(W ; \mathbb{Q})=0$, the homology exact sequence of the pair $(W, \partial W)$ gives $b_{1}(W)=0$, so it follows that $H_{*}(W ; \mathbb{Q}) \cong H_{*}\left(B^{4} ; \mathbb{Q}\right)$, and (1) holds. 
Now we show that (1) implies (3). Assume that Part (1) of the statement holds. It is a well-known fact that if $\frac{p}{q}=\left[a_{1}, \ldots, a_{n}\right]^{-}$the lens space $L(p, q)$ smoothly bounds the 4-dimensional plumbing $P(p, q)$ given by the weighted graph of Figure 8 . The

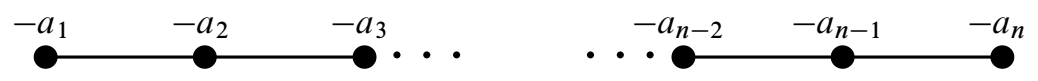

Figure 8: The graph of the canonical plumbing bounded by $L(p, q)$.

intersection form of $P(p, q)$ is negative definite. Hence, since $L(p, q) \cong-L(p, p-q)$, if $L(p, q)$ smoothly bounds a rational homology 4-ball $W(p, q)$ we can construct the smooth, negative 4-manifolds

$$
X(p, q)=P(p, q) \cup_{\partial}(-W(p, q)), \quad X(p, p-q)=P(p, p-q) \cup_{\partial} W(p, q) .
$$

By Donaldson's theorem on the intersection form of definite 4-manifolds [4], the intersection forms of $X(p, q)$ and $X(p, p-q)$ are both standard diagonal. Hence, suppose that the intersection lattice of $X(p, q)$ is isomorphic to $\mathbf{D}^{n}$ and the intersection lattice of $X(p, p-q)$ is isomorphic to $\mathbf{D}^{n^{\prime}}$. Clearly, the intersection lattices $H_{2}(P(p, q) ; \mathbb{Z}) \cong \mathbb{Z}^{n}$ and $H_{2}(P(p, p-q) ; \mathbb{Z}) \cong \mathbb{Z}^{n^{\prime}}$ have bases $\left\{v_{1}, \ldots, v_{n}\right\}$ and $\left\{w_{1}, \ldots, w_{n^{\prime}}\right\}$ which satisfy (3-1). Therefore, via the embeddings $P(p, q) \subset X(p, q)$ and $P(p, p-q) \subset X(p, p-q)$ we can view the above bases as standard subsets $S \subset \mathbf{D}^{n}$ and $S^{\prime} \subset \mathbf{D}^{n^{\prime}}$ with associated strings $\left(a_{1}, \ldots, a_{n}\right)$ and $\left(b_{1}, \ldots, b_{n^{\prime}}\right)$, where $\left[b_{1}, \ldots, b_{n^{\prime}}\right]^{-}=p /(p-q)$. In view of Lemma 2.6, we may assume without loss of generality that $I(S)<0$. Then, by Theorem 6.4 and Lemma 2.4, Lemma 9.2, Lemma 9.3 and Lemma 9.4 it follows that (3) holds.

Finally, we show that (3) implies (2). Suppose that (3) holds, ie $\frac{p}{q} \in \mathcal{R}$. Then, since applying finitely many times the functions $f$ and $g$ of Definition 1.1 amounts to changing $K(p, q)$ by an isotopy or a reflection, we may assume that $p=m^{2}$ and $q$ is of one of the three types given in Definition 1.1. We consider various cases separately.

First case $(q=m k \pm 1$, with $m>k>0$ and $(m, k)=1) \quad$ In view of Lemma 7.1 and Lemma 8.1, it suffices to show that the string of coefficients of the continued fraction expansion of $\frac{p}{q}$ is obtained from $(2,2,2)$ via a finite sequence of operations as in Lemma 7.1. Since $m^{2}-(m k \mp 1)=m(m-k) \pm 1$ and either $m \geq 2 k$ or $m \geq 2(m-k)$ up to replacing $k$ with $m-k$ (and $K(p, q)$ with its mirror image $K(p, p-q)$ ) we may assume $m \geq 2 k$. If $m=2 k$, since $(m, k)=1$ we must have $m=2, k=1$ and $p / q=[2,2,2]^{-}$. If $m>2 k$, arguing by induction on $m$ we may assume

$$
\frac{(m-k)^{2}}{(m-k) k \pm 1}=\left[a_{1}, a_{2}, \ldots, a_{n}\right]^{-},
$$


where $\left(a_{1}, a_{2}, \ldots, a_{n}\right)$ is obtained from $(2,2,2)$ as described above. But in view of Lemma 9.1 we have

$$
\frac{m^{2}}{m k \pm 1}=\left[a_{1}+1, a_{2}, \ldots, a_{n}, 2\right]^{-},
$$

so we are done.

Second case $(q=d(m-1)$, where $d>1$ divides $2 m+1) \quad$ It suffices to show that (2) holds for $K(p, p-q)$. Since $d(m-1)<m^{2}$, we have $2 m+1>d>1$, and $d$ must be odd because it divides $2 m+1$. Therefore we can write $d=2 s+3$ for some $s \geq 0$ and $2 m+1=d(2 t+3)$ for some $t \geq 0$. Then $m=2 s t+3 s+3 t+4$, and as in the proof of Lemma 9.3

$$
\frac{m^{2}}{m^{2}-d(m-1)}=\left[2^{[t]}, 3, s+2, t+2,3,2^{[s]}\right]^{-} .
$$

Therefore (2) holds by Lemma 8.4(1).

Third case $(q=d(m+1)$, where $d>1$ divides $2 m-1) \quad$ Arguing as in the previous case, we can write $d=2 s+3$ and $2 m-1=d(2 t+3)$ for some $s, t \geq 0$. Then, $m=2 s t+3 s+3 t+5$ and

$$
\frac{m^{2}}{d(m+1)}=\left[t+2,2, s+3,2^{[t]}, 4,2^{[s]}\right]^{-},
$$

which implies (2) by Lemma 8.5(2).

Fourth case $(q=d(m+1)$, where $d>1$ is odd and divides $m+1) \quad$ Since $d(m+1)<$ $m^{2}$ we have $m+1>d>1$, therefore we can write $d=2 s+3$ and $m+1=d(t+2)$ for some $s, t \geq 0$. Then

$$
\frac{m^{2}}{d(m+1)}=\left[t+2, s+2,3,2^{[t]}, 4,2^{[s]}\right]^{-},
$$

and (2) holds by Lemma 8.5(1).

Fifth case $(q=d(m-1)$, where $d>1$ is odd and divides $m-1) \quad$ As before, it suffices to prove that (2) holds for $K(p, p-q)$. We can write $d=2 s+3$ and $m-1=d(t+1)$ for some $s, t \geq 0$. Then

$$
\frac{m^{2}}{m^{2}-d(m-1)}=\left[2^{[t]}, s+3,2, t+2,3,2^{[s]}\right]^{-},
$$

and (2) holds by Lemma 8.4(2). This concludes the proof.

\section{References}

[1] G Burde, H Zieschang, Knots, de Gruyter Studies in Mathematics 5, Walter de Gruyter \& Co., Berlin (1985) MR808776 
[2] A J Casson, CM Gordon, Cobordism of classical knots, from: "À la recherche de la topologie perdue", Progr. Math. 62, Birkhäuser, Boston (1986) 181-199 MR900252With an appendix by P. M. Gilmer

[3] A J Casson, J L Harer, Some homology lens spaces which bound rational homology balls, Pacific J. Math. 96 (1981) 23-36 MR634760

[4] S K Donaldson, The orientation of Yang-Mills moduli spaces and 4-manifold topology, J. Differential Geom. 26 (1987) 397-428 MR910015

[5] R Fintushel, R Stern, Rational homology cobordisms of spherical space forms, Topology 26 (1987) 385-393 MR899056

[6] P Orlik, P Wagreich, Algebraic surfaces with $k^{*}$-action, Acta Math. 138 (1977) 43-81 MR0460342

[7] B Owens, S Strle, Rational homology spheres and the four-ball genus of knots, Adv. Math. 200 (2006) 196-216 MR2199633

[8] P Ozsváth, Z Szabó, Absolutely graded Floer homologies and intersection forms for four-manifolds with boundary, Adv. Math. 173 (2003) 179-261 MR1957829

[9] P Popescu-Pampu, The geometry of continued fractions and the topology of surface singularities (2005) arXiv:math.GT/0506432 To appear in Singularities-Sapporo 2004, Advanced Studies in Pure Mathematics, Kinokuniya, Tokyo 2006

[10] O Riemenschneider, Deformationen von Quotientensingularitäten (nach zyklischen Gruppen), Math. Ann. 209 (1974) 211-248 MR0367276

[11] L Siebenman, Exercises sur les noeuds rationnels (1975) mimeographed notes, Orsay

Dipartimento di Matematica “L. Tonelli”, Largo Bruno Pontecorvo, 5, Università di Pisa I-56127 Pisa, ITALY

lisca@dm.unipi.it

Proposed: Cameron Gordon

Seconded: Peter Ozsvath and Tom Mrowka

Received: 12th June 2006

Accepted: 18th January 2007 